tend to be very unstable and to exhibit a poor out-of-sample performance. Furthermore, the classical model implicitly assumes that returns follow a normal distribution or that investors have a quadratic utility function.

It is known that the distribution of the returns exhibits fatter tails than the Gaussian distribution (see, e.g., Mandelbrot, 1963; Turner and Weigel, 1992). Arditti (1967), Kraus and Litzenberger (1976) and Harvey and Siddique (2000) suggest that the investor's utility function is not quadratic, since investors have preference for positive skewness. In fact, there are several studies suggesting gains if, instead of considering just the first two moments of the returns' distribution, higher moments are also included in the portfolio management problem (Chunhachinda et al., 1997; Mencia and Sentana, 2009; Harvey et al., 2010).

With the aim of improving the performance of the classical mean-variance model, several modifications or alternative strategies have been proposed. One well-known modification consists in imposing a cardinality constraint (a constraint that limits the number of stocks in the portfolio) to the classical mean-variance model, leading to the cardinality constrained meanvariance model. This model can be seen as a mixed-integer quadratic problem and is no longer solved in polynomial time. In the literature, several algorithms have been proposed to solve this cardinality constrained portfolio management problem.

This paper focus on the analysis of the cardinality impact on the portfolio performance, in the mean-variance (hereafter MV), the mean-variance-skewness (MVS) and the mean-varianceskewness-kurtosis (MVSK) frameworks. Therefore, the analysis is conducted not only on the impact of the cardinality constraint in each framework but also on the benefits of considering higher moments in portfolio management. In this study we assume that the investor maximizes her expected utility, characterized by constant relative risk aversion preferences (CRRA). This is a common assumption in the related literature (see, e.g., Aitt-Sahalia and Brandt, 2001). The main reason why we have adopted this particular utility function is the same one that is stated by Brandt et al. (2009), p. 3421: "the advantage of CRRA utility is that it incorporates preferences toward higher moments in a parsimonious manner. In addition, the utility function is twice continuously differentiable, which allows us to use more efficient numerical optimization algorithms that make use of the analytic gradient and Hessian of the objective function". Nevertheless, we point out that the presented procedures are quite flexible, and may be applied to any other type of utility function. For each framework, we propose a bi-objective model that allows the investor to directly analyse the efficient tradeoff between expected utility and cardinality. Given the non-smoothness of the cardinality function and inspired in Brito and Vicente (2014), we have decided to solve each bi-objective model using a derivative-free solver based on direct multisearch (Custódio et al., 2011).

The contribution of this paper is twofold. First, this study extends the analysis of the cardinality impact on the portfolio performance from the standard MV framework to the MVS and MVSK frameworks. Second, we analyse the performance gains (in terms of certainty equivalent and Sharpe ratio) when considering higher moments (skewness and kurtosis) at different cardinality levels. The certainty equivalent, being a measure that takes into account the expected utility, is therefore a more suitable performance measure than the Sharpe ratio when comparing the three frameworks. Although one needs to be careful when using the Sharpe ratio as a performance measure, especially in the MVS and MVSK frameworks (the ratio does not account for skewness or kurtosis), this is one of the most widely used performance measures in the literature, even in non mean-variance settings (see, e.g., DeMiguel et al., 2009). Although the Sharpe ratio is intrinsically related to the MV framework, it is interesting to see how the 
MVS and MVSK frameworks perform in this dimension in comparison with the MV framework (even if the existence of performance gains, in terms of Sharpe ratio, of the MVS and MVSK frameworks against the MV framework should be hard to get).

The empirical analysis is conducted on a dataset from the Portuguese Stock Index (PSI20 index). The daily data is collected from Thomson Reuters Datastream ${ }^{\circledR}$. First, we compute the expected utility/cardinality efficient frontiers for each one of the three frameworks and analyse the in-sample cardinality impact using as performance measures the certainty equivalent and the Sharpe ratio. Second, we perform an out-of-sample analysis using the certainty equivalent, the Sharpe ratio, the turnover and the Sharpe ratio of returns net of transaction costs. The results for each efficient expected utility/cardinality portfolio in each of the three frameworks are reported.

The in-sample results show that both the certainty equivalent and the Sharpe ratio increase with the cardinality level and suggest that there are no gains, in terms of certainty equivalent, when considering higher moments. When transaction costs are not considered, the out-of-sample results on the certainty equivalent and Sharpe ratio do not show a clear trend with cardinality. However, the turnover increases up to a certain level of cardinality and then decreases. This result leads to another interesting result that the out-of-sample net Sharpe ratio - that is, the Sharpe ratio net of transaction costs - decreases up to a certain cardinality level, then increases and afterwards behaves more erratically for larger values of the cardinality. So, for an important range of cardinality levels, diversification has a positive effect when transaction costs are taken into account.

The out-of-sample certainty equivalent and the Sharpe ratio suggest that there are gains, for certain cardinality levels, when considering skewness and kurtosis (although these differences were not statistically significant). In the case of the net Sharpe ratio and when considering skewness, there statistical significant gains for certain cardinality levels.

Finally, as a kind of robustness check, we present some results for the out-of-sample certainty equivalent, Sharpe ratio, turnover and the net Sharpe ratio of a large dataset from the EUROSTOXX50 index. The observed results are consistent with those for the PSI20 index. However, since in this dataset we have a larger number of stocks, we are able to build portfolios with a larger cardinality. We notice that, for larger levels of cardinality, the turnover and the net Sharpe ratio no longer show a clear trend. After an initial increase in turnover, and the subsequent decrease, the turnover increases again and tends to behave more erratically. Similarly, after an initial decrease in the net Sharpe ratio, and the subsequent increase, the net Sharpe ratio decreases again and tends to behave more erratically. This indicates that the best level of cardinality should be a concern for investors wanting to diversify their portfolios, and that we cannot assume that a larger cardinality is always better.

The remainder of the paper proceeds as follows. We present a brief literature review in Section 2. In Section 3 we describe our approach and the proposed models. The empirical application is presented in Section 4. We conclude in Section 5.

\section{State of the Art}

At least since the works of Mandelbrot (1963) and Fama (1965), it is well documented that one of the stylized facts of financial series is that the distribution of the returns exhibits fat tails. This fact was explored in major papers associated to ARCH type (e.g., Engle, 1982; Bollerslev, 1986; 
Nelson, 1991; Zakoian, 1994; Tavares et al., 2008) and stochastic volatility models (e.g., Taylor, 1986; Bai et al., 2003). Several studies have analysed this stylized fact for the specific case of the Portuguese Stock Index (PSI20 index), (see, e.g., Curto et al., 2003; Rege et al., 2013). In portfolio management, the use of just the first two moments of the returns distribution should be questioned once we know that investors have preference for positive skewness (Arditti, 1967, give us an analytical proof of this fact) and dislike high kurtosis (see, e.g., Maringer and Parpas, 2009). In fact, the sufficient conditions for using just the first two moments are the normal distribution of returns and a quadratic utility function of the investors. But both of these conditions are rarely verified, which suggests that the classical mean-variance analysis, proposed by Markowitz (1952), may not be the most suitable approach for portfolio management. Hence it seems that there may be real gains in portfolio management when higher moments are considered. There are several studies incorporating higher moments in portfolio management (Chunhachinda et al., 1997; Athayde and Flôres, 2004; Maringer and Parpas, 2009; Mencia and Sentana, 2009; Harvey et al., 2010), however, its usefulness has not been clearly proven. For example, Cremers et al. (2005) showed empirically that for an investor with log or power preferences there are no significant differences between the mean-variance model and the other models with higher moments.

Cardinality is a well-known measure of portfolio diversification. When cardinality increases, the total risk of a portfolio decreases gradually, until a certain level of risk is achieved. After that level, increases in cardinality only produce negligible reductions in the risk level (see, e.g., Evans and Archer, 1968; Statman, 1987; Benartzi and Thaler, 2001). Therefore, the cardinality impact on portfolio performance is a very important issue for both individual and institutional investors.

The starting point for the cardinality issue in portfolio management has been the classical MV model, with the inclusion of a cardinality constraint. This model can be seen as a mixedinteger quadratic problem that is no longer solved in polynomial time, therefore most studies have focused on providing efficient algorithms to solve this problem. These algorithms vary from exact algorithms (Bienstock, 1996; Vielma et al., 2008; Bertsimas and Shioda, 2009) to heuristics (Chang et al., 2000; Fieldsend et al., 2004; Cesarone et al., 2009; Anagnostopoulos and Mamanis, 2011; Woodside-Oriakhi et al., 2011; Brito and Vicente, 2014). With the aim of promoting regularization of ill conditioning, DeMiguel et al. (2009) constrained the Markowitz classical model by imposing a bound on the $l_{1}$-norm of the vector of portfolio weights, among other possibilities. Since the $l_{1}$-norm is the exact convex relaxation of the $l_{0}$-norm (cardinality), DeMiguel et al. (2009) indirectly studied the effect of cardinality in the classical MV framework. A similar idea was explored in Brodie et al. (2009).

In this paper we extend the study of the cardinality impact from the standard MV framework to the MVS and MVSK frameworks. For each framework, based on the investor's expected utility maximization criterion, we propose a bi-objective model that allows the investor to directly analyse the efficient tradeoff between expected utility and cardinality. Given the lack of derivatives of the cardinality function, and inspired by Brito and Vicente (2014), we apply a directional derivative-free algorithm (Custódio et al., 2011) to obtain the solution of the bi-objective optimization problems. 


\section{Methodology}

Suppose that an investor has a certain wealth, $W$, to invest in a set of $N$ stocks. Let $u(\cdot)$ be the investor's utility function. Assuming that the investor has CRRA (constant relative risk aversion) preferences, her utility function is given by

$$
u\left(r_{p, t+1}\right)=\frac{\left(1+r_{p, t+1}\right)^{1-\gamma}}{1-\gamma}, \text { with } \gamma>1,
$$

where $r_{p, t+1}$ is the portfolio return at time $t+1$ and $\gamma$ is the relative risk aversion coefficient (higher values of $\gamma$ imply more risk aversion).

Considering the fourth order Taylor expansion of the expected utility, $E_{t}\left[u\left(r_{p, t+1}\right)\right]$, around the expected return of the portfolio, $E_{t}\left(r_{p, t+1}\right)$, we have

$$
\begin{aligned}
E_{t}\left[u\left(r_{p, t+1}\right)\right] & \approx u\left[E_{t}\left(r_{p, t+1}\right)\right]+\frac{1}{2 !} u^{\prime \prime}\left[E_{t}\left(r_{p, t+1}\right)\right] V_{t}\left(r_{p, t+1}\right) \\
& +\frac{1}{3 !} u^{\prime \prime \prime}\left[E_{t}\left(r_{p, t+1}\right)\right] S_{t}\left(r_{p, t+1}\right) \\
& +\frac{1}{4 !} u^{\prime \prime \prime \prime}\left[E_{t}\left(r_{p, t+1}\right)\right] K_{t}\left(r_{p, t+1}\right)
\end{aligned}
$$

where

$$
\begin{aligned}
V_{t}\left(r_{p, t+1}\right) & =E_{t}\left[r_{p, t+1}-E_{t}\left(r_{p, t+1}\right)\right]^{2}, \\
S_{t}\left(r_{p, t+1}\right) & =E_{t}\left[r_{p, t+1}-E_{t}\left(r_{p, t+1}\right)\right]^{3}, \\
K_{t}\left(r_{p, t+1}\right) & =E_{t}\left[r_{p, t+1}-E_{t}\left(r_{p, t+1}\right)\right]^{4},
\end{aligned}
$$

are the portfolio variance, skewness and kurtosis, respectively. Since it is assumed that the investor has CRRA preferences (with $\gamma>1$ ), we have

$$
\begin{aligned}
u\left[E_{t}\left(r_{p, t+1}\right)\right] & =\frac{\left[1+E_{t}\left(r_{p, t+1}\right)\right]^{1-\gamma}}{1-\gamma}, \\
u^{\prime \prime}\left[E_{t}\left(r_{p, t+1}\right)\right] & =-\gamma\left[1+E_{t}\left(r_{p, t+1}\right)\right]^{-(\gamma+1)}, \\
u^{\prime \prime \prime}\left[E_{t}\left(r_{p, t+1}\right)\right] & =\gamma(\gamma+1)\left[1+E_{t}\left(r_{p, t+1}\right)\right]^{-(\gamma+2)}, \\
u^{\prime \prime \prime \prime}\left[E_{t}\left(r_{p, t+1}\right)\right] & =-\gamma(\gamma+1)(\gamma+2)\left[1+E_{t}\left(r_{p, t+1}\right)\right]^{-(\gamma+3)} .
\end{aligned}
$$


Now, for the sake of notation simplicity, let us consider that

$$
\begin{aligned}
a & =u\left[E_{t}\left(r_{p, t+1}\right)\right], \\
b & =-\frac{u^{\prime \prime}\left[E_{t}\left(r_{p, t+1}\right)\right]}{2}, \\
c & =\frac{u^{\prime \prime \prime}\left[E_{t}\left(r_{p, t+1}\right)\right]}{6}, \\
d & =-\frac{u^{\prime \prime \prime \prime}\left[E_{t}\left(r_{p, t+1}\right)\right]}{24} .
\end{aligned}
$$

Then Equation (2) can be rewritten as

$$
E_{t}\left[u\left(r_{p, t+1}\right)\right] \approx a-b V_{t}\left(r_{p, t+1}\right)+c S_{t}\left(r_{p, t+1}\right)-d K_{t}\left(r_{p, t+1}\right)
$$

\subsection{The Expected Utility/Cardinality Bi-objective Model}

We assume that the investor wants to choose a portfolio taking into account two criteria: an expected utility that considers several moments of the portfolio return and the cardinality of the portfolio.

The problem of maximizing the expected utility can be stated as the choice of the portfolio weights, $w_{i}$, that maximize the conditional expected utility of the portfolio's return, $r_{p, t+1}$,

$$
\begin{aligned}
\max _{w \in \mathbb{R}^{N}} & E_{t}\left[u\left(r_{p, t+1}\right)\right]=E_{t}\left[u\left(\sum_{i=1}^{N} w_{i} r_{i, t+1}\right)\right] \\
\text { subject to } & e^{\top} w=1, \\
& L_{i} \leq w_{i} \leq U_{i}, i=1, \ldots, N
\end{aligned}
$$

where $e$ is the $N \times 1$ vector of ones, and $L_{i}$ and $U_{i}$ represent, respectively, lower and upper bounds on the weights.

Adding to Problem (4) a constraint that limits the number of active positions on the portfolio (a cardinality constraint), we have

$$
\begin{aligned}
\max _{w \in \mathbb{R}^{N}} & E_{t}\left[u\left(r_{p, t+1}\right)\right]=E_{t}\left[u\left(\sum_{i=1}^{N} w_{i} r_{i, t+1}\right)\right] \\
\text { subject to } & \operatorname{card}(w) \leq K \\
& e^{\top} w=1 \\
& L_{i} \leq w_{i} \leq U_{i}, i=1, \ldots, N
\end{aligned}
$$

where $\operatorname{card}(w)=\left|\left\{i \in\{1, \ldots, N\}: w_{i} \neq 0\right\}\right|$ and $K \in\{1, \ldots, N\}$. Despite $\operatorname{card}(x)$ being often called, in the literature, the $l_{0}$-norm, it is not a norm due to the fact that it doesn't satisfy the homogeneity property.

Here we suggest that cardinality should be included as a second objective in the problem, instead of a constraint. Thus, we reformulate Problem (5) as 


$$
\begin{aligned}
\max _{w \in \mathbb{R}^{N}} & E_{t}\left[u\left(r_{p, t+1}\right)\right]=E_{t}\left[u\left(\sum_{i=1}^{N} w_{i} r_{i, t+1}\right)\right] \\
\min _{w \in \mathbb{R}^{N}} & \operatorname{card}(w) \\
\text { subject to } & e^{\top} w=1, \\
& L_{i} \leq w_{i} \leq U_{i}, i=1, \ldots, N .
\end{aligned}
$$

The solution of Problem (6) is given by a Pareto front, i.e., as an efficient expected utility/cardinality frontier. Efficient expected utility/cardinality portfolios can be seen as those which have the maximum expected utility among all that have at most a certain level of cardinality. The investor can thus directly analyse the efficient tradeoff between expected utility and cardinality. The bi-objective Problem (6) has two objective functions, a linear constraint and $2 N$ inequality constraints. The first objective, $E_{t}\left[u\left(r_{p, t+1}\right)\right]$, is nonlinear but smooth. However, the second objective, $\operatorname{card}(w)$, is a piecewise linear discontinuous function, and consequently it is nonlinear and non-smooth. Since derivative-free algorithms are applicable to black-box type functions (see Conn et al., 2009, for a survey on derivative-free optimization), they are suitable to deal with the cardinality objective function. According with Custódio et al. (2011), direct multisearch has the best performance among all derivative-free solvers for multiobjective optimization. We have thus decided to solve Problem (6) using the DMS solver (version 0.3) ${ }^{1}$.

Taking into account Problem (6), we suggest to analyse separately the impact of cardinality on the portfolio performance for three different frameworks (see Section 3.2): when the investor, with CRRA preferences, has MV, MVS and MVSK expected utilities.

\subsection{The MV, MVS and MVSK Expected Utility/Cardinality Efficient Fron- tiers}

If the investor with CRRA preferences (see Equation (1)) considers the second order Taylor expansion of the expected utility, $E_{t}\left[u\left(r_{p, t+1}\right)\right]$, around the expected return of the portfolio, $E_{t}\left(r_{p, t+1}\right)$, the second member of Equation (3) is truncated at the second term. Hence, in order to investigate the cardinality impact, the following model can be solved when the investor has a MV expected utility

$$
\begin{array}{rl}
\max _{w \in \mathbb{R}^{N}} & a-b V_{t}\left(r_{p, t+1}\right) \\
\min _{w \in \mathbb{R}^{N}} & \operatorname{card}(w) \\
\text { subject to } & e^{\top} w=1, \\
& L_{i} \leq w_{i} \leq U_{i}, i=1, \ldots, N
\end{array}
$$

By solving Problem (7), we identify a MV expected utility/cardinality efficient frontier. A portfolio in this frontier is such that there exists no other feasible one which simultaneously presents a higher expected MV utility and a lower cardinality. Given such an efficient frontier and a cardinality target, an investor may directly find the answers to what is the optimal (highest) expected utility level that can be attained while meeting the cardinality target and what are the portfolios leading to such an expected utility level. The investor can thus directly examine the efficient tradeoff between the MV expected utility and cardinality.

\footnotetext{
${ }^{1}$ The DMS solver is available, by request, at the site: http://www.mat.uc.pt/dms/.
} 
If the investor, besides the mean and variance, also considers the third moment (the second member of Equation (3) is truncated at the third term), the following model can be solved

$$
\begin{array}{rl}
\max _{w \in \mathbb{R}^{N}} & a-b V_{t}\left(r_{p, t+1}\right)+c S_{t}\left(r_{p, t+1}\right) \\
\min _{w \in \mathbb{R}^{N}} & \operatorname{card}(w) \\
\text { subject to } & e^{\top} w=1, \\
& L_{i} \leq w_{i} \leq U_{i}, i=1, \ldots, N,
\end{array}
$$

By solving Problem (8), we identify a MVS expected utility/cardinality efficient frontier. The investor can thus directly analyse the efficient tradeoff between the MVS expected utility and cardinality.

When the investor considers all the moments present in Equation (3), the model to be solved is given by

$$
\begin{array}{rl}
\max _{w \in \mathbb{R}^{N}} & a-b V_{t}\left(r_{p, t+1}\right)+c S_{t}\left(r_{p, t+1}\right)-d K_{t}\left(r_{p, t+1}\right) \\
\min _{w \in \mathbb{R}^{N}} & \operatorname{card}(w) \\
\text { subject to } & e^{\top} w=1, \\
& L_{i} \leq w_{i} \leq U_{i}, i=1, \ldots, N,
\end{array}
$$

By solving Problem (9), we identify a MVSK expected utility/cardinality efficient frontier. The investor can thus directly analyse the efficient tradeoff between the MVSK expected utility and cardinality.

\section{Empirical performance}

We tested the proposed methodology in one dataset based on the composition of the Portuguese Stock Index (PSI20 index). We collected daily data from Thomson Reuters Datastream ${ }^{\circledR}$, for the time window from July 2007 to June 2014 (seven years). We chose 20 stocks of companies that belonged to the PSI20 index at least once and that were traded during all this time (some stocks changed designation, but all stocks were quoted during the entire period under analysis). The composition of this dataset is given in Table 1 . 
Table 1: The PSI20 dataset

\begin{tabular}{l|l}
\hline \multicolumn{2}{c}{ List of stocks } \\
\hline ALTRI SGPS & MARTIFER \\
BANCO BPI & MEDIA CAPITAL \\
BANCO COMR.PORTUGUES 'R' & MOTA ENGIL SGPS \\
BANCO ESPIRITO SANTO & NOS SGPS \\
COFINA & NOVABASE \\
EDP ENERGIAS DE PORTUGAL & PHAROL SGPS \\
GI.GLB.INTEL.TECHS.SGPS & PORTUCEL EMPRESA \\
IMPRESA SGPS & SEMAPA \\
INAPA & SONAE INDUSTRIA SGPS \\
JERONIMO MARTINS & SONAE SGPS \\
\hline
\end{tabular}

This table lists the composition of the PSI20 dataset used in the empirical work. Daily closing prices, from July 2007 to June 2014, of these 20 stocks were collected from the Thomson Reuters Datastream ${ }^{\circledR}$.

Based on the daily closing prices, $p_{i, t}$, we computed the discrete daily returns,

$$
r_{i, t}=\frac{p_{i, t}-p_{i, t-1}}{p_{i, t-1}}, \quad i=1, \ldots, N, \quad t=1, \ldots, T,
$$

where $N$ is the number of stocks $(N=20)$ and $T$ is the number of observations $(T=1827)$. Some descriptive statistics of the returns, for the overall sample period, are reported in Table 2 .

Table 2: Descriptive statistics for the PSI20 dataset

\begin{tabular}{r|r}
\hline \hline Number of days & 1826 \\
\hline Minimum & -0.1573 \\
\hline Median & -0.0001 \\
\hline Maximum & 0.2584 \\
\hline Mean & -0.0002 \\
\hline Variance & 0.0009 \\
\hline Skewness & 0.8187 \\
\hline Kurtosis & 15.6667
\end{tabular}

This table reports some descriptive statistics for the PSI20 dataset (the composition of the dataset is reported in Table 1). These statistics are averaged cross-sectionally, i. e., they are computed for each stock and then the arithmetic mean is taken.

During the period under scrutiny the discrete daily returns of the PSI20 stocks presented, on average, a negative but near zero mean, positive skewness and about five times above normal kurtosis.

We present the efficient expected utility/cardinality portfolios, with the expected utility based on the MV, MVS and MVSK frameworks, in Section 4.1. In Section 4.2, based on a rolling-sample approach, we analyse the out-of-sample performance of these efficient portfolios. 
Both in-sample and out-of-sample analysis were conducted assuming that the investor has CRRA preferences and a parameter of relative risk aversion equal to five (this is a common assumption, see, e.g., Brandt et al., 2009).

\subsection{In-sample performance}

We solved Problem (7), Problem (8) and Problem (9) for the general non-boundary case (i.e., $L_{i}=-\infty$ and $U_{i}=+\infty$ ) using the DMS solver (version 0.3$)^{2}$. Other choices for the boundaries could be made; for instance, if short selling is undesirable then $L_{i} \geq 0$ and consequently $U_{i} \leq 1$. We have decided to allow for short selling in order to make the models as general as possible and to avoid the possibility of getting Pareto frontiers with few feasible cardinality levels. Note that the greater the restriction on the portfolio weights, the more likely it is to obtain Pareto frontiers without all the feasible cardinality levels (these "missing points" correspond to strictly dominated points $)^{3}$. Allowing for short selling in each model does not make them less realistic, since most markets around the world allow short positions and, particularly, the Portuguese Securities Market Commission (CMVM) allows to perform short selling in each one of the constituents of the PSI20 index.

In the in-sample analysis we used the overall sample period, from July 2007 to June 2014 (seven years). The efficient expected utility/cardinality frontiers ${ }^{4}$, for the MV, MVS and MVSK frameworks, are plotted in Figure 1. Direct multisearch aims to approximate the true Pareto frontier. Theoretically it is only possible to prove that there is a limit point in a stationary form of the Pareto frontier, as no aggregation or scalarization technique is incorporated (see Custódio et al., 2011, for further details). However, some empirical applications have shown that this method has a very good performance, even when applied to problems with discontinuous and non-convex Pareto frontiers ( Custódio et al., 2011). In our application, we can observe that direct multisearch was effective, in the sense that it allowed us to obtain frontiers for the three frameworks, and we have all the reasons to believe that they are good approximations to the true Pareto frontiers. From the analysis of these frontiers the investor can thus directly analyse the efficient tradeoff (in each framework) and choose (according to some pre-established criteria), in the expected utility/cardinality space, the portfolio that best fits her preferences.

\footnotetext{
${ }^{2}$ Following Chang et al. (2000) and Jobst et al. (2010), in practice the true cardinality is approximated by introducing a tolerance $\varepsilon=1 \%$, such that $\operatorname{card}(w)=\sum_{i=1}^{N} 1_{\left\{\left|w_{i}\right|>\varepsilon\right\}}$, where11 represents the indicator function.

${ }^{3}$ Theoretically the solutions of Problem (7), Problem (8) and Problem (9) can be a single point that strictly dominates all the others.

${ }^{4}$ Note that, with the $1 \%$ threshold for the cardinality computation, the maximum cardinality level that led to non-dominated solutions was 16 .
} 


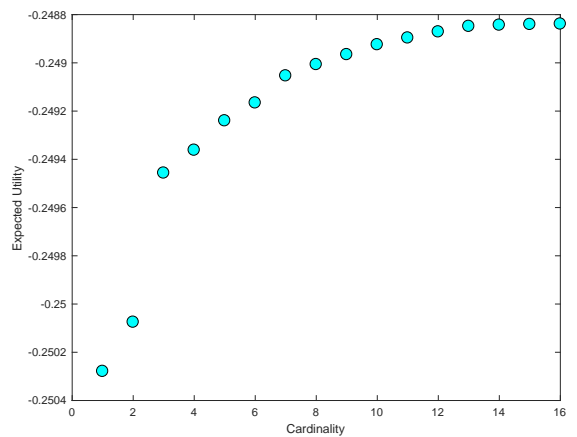

Efficient MV expected utility/cardinality portfolios

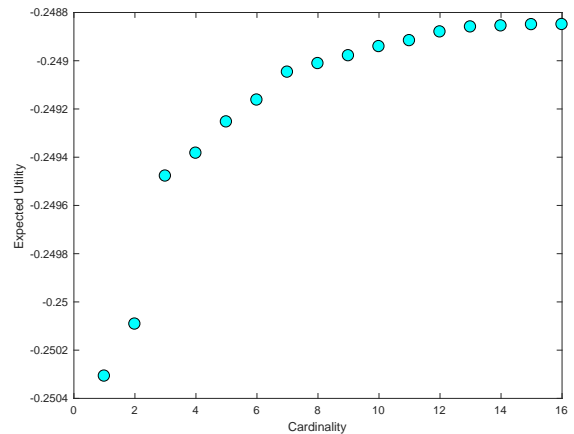

Efficient MVSK expected utility/cardinality portfolios

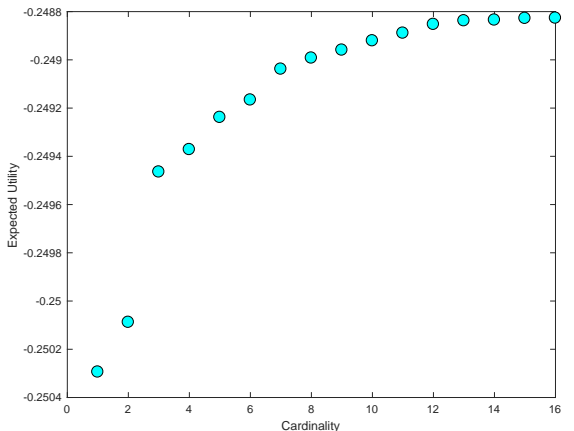

Efficient MVS expected utility/cardinality portfolios

Figure 1: Efficient expected utility/cardinality frontiers, for the MV, MVS and MVSK frameworks

Table 3 reports the certainty equivalent return for the MV, MVS and MVSK frameworks. The certainty equivalent return, $\widehat{C E}$, is defined as

$$
U(\widehat{C E})=E U(\hat{\mu}),
$$

where $\hat{\mu}$ denotes the portfolio mean and $E U($.$) represents the expected utility defined in Equa-$ tion (3). The certainty equivalent can be interpreted as the risk-free rate that an investor is willing to accept in order to give up a particular risky investment. 
Table 3: In-sample certainty equivalent return

\begin{tabular}{c|r|r|r}
\hline \multicolumn{4}{c}{ Efficient portfolios } \\
\hline \hline Number of stocks & MV & MVS & MVSK \\
\hline 1 & -0.2499 & -0.2637 & -0.2762 \\
2 & $-0,0171$ & -0.0245 & -0.0280 \\
3 & 0.5494 & 0.5498 & 0.5365 \\
4 & 0.6475 & 0.6437 & 0.6340 \\
5 & 0.7735 & 0.7814 & 0.7661 \\
6 & 0.8469 & 0.8516 & 0.8536 \\
7 & 0.9543 & 0.9694 & 0.9638 \\
8 & 0.9970 & 1.0127 & 0.9936 \\
9 & 1.0596 & 1.0457 & 1.0256 \\
10 & 1.0976 & 1.1020 & 1.0795 \\
11 & 1.1279 & 1.1319 & 1.1074 \\
12 & 1.1626 & 1.1694 & 1.1463 \\
13 & 1.1815 & 1.1846 & 1.1623 \\
14 & 1.1859 & 1.1889 & 1.1665 \\
15 & 1.1905 & 1.1930 & 1.1709 \\
16 & 1.1956 & 1.1989 & 1.1760 \\
\hline
\end{tabular}

This table lists the in-sample certainty equivalent return $\widehat{(C E})$ of the efficient expected utility/cardinality portfolios. $M V$ refers to the efficient MV expected utility/cardinality portfolios, MVS refers to the efficient MVS expected utility/cardinality portfolios and MVSK refers to the efficient MVSK expected utility/cardinality portfolios. All the certainty equivalent values are multiplied by a factor of 1000 .

Tables 4, 5 and 6 report some descriptive statistics (the in-sample mean, $\hat{\mu}$, standard deviation, $\hat{\sigma}$, skewness, $\hat{\xi}$, kurtosis, $\hat{\kappa}$, expected utility, $\hat{U}$, and Sharpe ratio, $\hat{S}$ ) for the MV, MVS and MVSK frameworks, respectively. These tables also report the ratio between the in-sample Sharpe ratio of each efficient portfolio and the in-sample Sharpe ratio of the efficient portfolio with cardinality $16, \hat{S} / \hat{S}_{16}$ (corresponding to the portfolio with the maximum cardinality level). This ratio can be seen as a measure of the impact of portfolio diversification on the Sharpe ratio. Given the time series of daily returns, for each portfolio, the Sharpe ratio is defined as the sample mean of excess returns (over the risk-free asset ${ }^{5}$ ), $\hat{\mu}$, divided by its sample standard deviation, $\hat{\sigma}$ :

$$
\hat{S}=\frac{\hat{\mu}}{\hat{\sigma}} .
$$

\footnotetext{
${ }^{5}$ Without loss of generality, in this paper we set $r_{f}=0$.
} 
Table 4: In-sample analysis of the efficient MV expected utility/cardinality portfolios

\begin{tabular}{c|ccccccc}
\hline \multicolumn{10}{c}{} & \multicolumn{7}{c}{ Efficient portfolios } \\
\hline \hline Number of stocks & $\hat{\mu}$ & $\hat{\sigma}$ & $\hat{\xi}$ & $\hat{\kappa}$ & $\hat{U}$ & $\hat{S}$ & $\hat{S} / \hat{S}_{16}$ \\
\hline 1 & 0.0008 & 0.0204 & -0.3440 & 8.4078 & -0.2503 & 0.0385 & $0.3485(0.0038)$ \\
2 & 0.0007 & 0.0164 & -0.3867 & 7.5859 & -0.2500 & 0.0398 & $0.3593(0.0036)$ \\
3 & 0.0019 & 0.0233 & -0.1037 & 5.2381 & -0.2495 & 0.0816 & $0.7373(0.0486)$ \\
4 & 0.0019 & 0.0225 & -0.1122 & 5.3278 & -0.2494 & 0.0847 & $0.7659(0.0556)$ \\
5 & 0.0023 & 0.0248 & -0.0292 & 4.8302 & -0.2492 & 0.0929 & $0.8394(0.1356)$ \\
6 & 0.0023 & 0.0238 & -0.0045 & 5.3832 & -0.2492 & 0.0948 & $0.8566(0.1480)$ \\
7 & 0.0025 & 0.0252 & 0.1553 & 6.1148 & -0.2490 & 0.1005 & $0.9081(0.2685)$ \\
8 & 0.0025 & 0.0243 & 0.1966 & 6.3847 & -0.2490 & 0.1014 & $0.9164(0.2531)$ \\
9 & 0.0027 & 0.0256 & 0.0374 & 5.8750 & -0.2489 & 0.1049 & $0.9484(0.4015)$ \\
10 & 0.0028 & 0.0259 & 0.0250 & 5.7805 & -0.2489 & 0.1067 & $0.9644(0.4727)$ \\
11 & 0.0029 & 0.0265 & 0.0159 & 5.7081 & -0.2489 & 0.1083 & $0.9792(0.6155)$ \\
12 & 0.0029 & 0.0263 & 0.0460 & 5.5404 & -0.2488 & 0.1094 & $0.9893(0.7229)$ \\
13 & 0.0029 & 0.0261 & 0.0054 & 5.5654 & -0.2488 & 0.1100 & $0.9946(0.7778)$ \\
14 & 0.0029 & 0.0261 & 0.0037 & 5.5670 & -0.2488 & 0.1102 & $0.9963(0.8238)$ \\
15 & 0.0029 & 0.0262 & 0.0105 & 5.6330 & -0.2488 & 0.1105 & $0.9986(0.9426)$ \\
16 & 0.0029 & 0.0261 & 0.0144 & 5.4855 & -0.2488 & 0.1106 & 1 \\
\hline
\end{tabular}

This table reports the in-sample mean $(\hat{\mu})$, standard deviation $(\hat{\sigma})$, skewness $(\hat{\xi})$, kurtosis $(\hat{\kappa})$, Expected Utility $(\hat{U})$ and the in-sample Sharpe ratio $(\hat{S})$ of each efficient $M V$ expected utility/cardinality portfolio. It also reports the ratio, $\hat{S} / \hat{S}_{16}$, between the in-sample Sharpe ratio of each efficient portfolio and the in-sample Sharpe ratio of the efficient portfolio with cardinality 16 (the highest Sharpe ratio portfolio). The Ledoit and Wolf (2008) bootstrap $p$-values for the statistical significance of the difference between the in-sample Sharpe ratio of the efficient portfolio with cardinality 16 and the respective efficient portfolio are presented in parenthesis. 
Table 5: In-sample analysis of the efficient MVS expected utility/cardinality portfolios

\begin{tabular}{c|ccccccc}
\hline \multicolumn{10}{c}{} & \multicolumn{7}{c}{ Efficient portfolios } \\
\hline \hline Number of stocks & $\hat{\mu}$ & $\hat{\sigma}$ & $\hat{\xi}$ & $\hat{\kappa}$ & $\hat{U}$ & $\hat{S}$ & $\hat{S} / \hat{S}_{16}$ \\
\hline 1 & 0.0008 & 0.0204 & -0.3441 & 8.4074 & -0.2503 & 0.0385 & $0.3486(0.0034)$ \\
2 & 0.0006 & 0.0163 & -0.3851 & 7.5589 & -0.2500 & 0.0396 & $0.3584(0.0040)$ \\
3 & 0.0019 & 0.0232 & -0.1021 & 5.2614 & -0.2495 & 0.0817 & $0.7395(0.0620)$ \\
4 & 0.0019 & 0.0224 & -0.1103 & 5.3212 & -0.2494 & 0.0847 & $0.7661(0.0734)$ \\
5 & 0.0023 & 0.0246 & -0.0158 & 4.8868 & -0.2492 & 0.0929 & $0.8408(0.1476)$ \\
6 & 0.0023 & 0.0239 & -0.0035 & 5.3673 & -0.2492 & 0.0950 & $0.8596(0.1672)$ \\
7 & 0.0026 & 0.0254 & 0.2250 & 6.7244 & -0.2490 & 0.1005 & $0.9095(0.2589)$ \\
8 & 0.0025 & 0.0245 & 0.2437 & 6.8288 & -0.2490 & 0.1015 & $0.9180(0.2599)$ \\
9 & 0.0026 & 0.0251 & 0.2110 & 6.3837 & -0.2490 & 0.1033 & $0.9349(0.3155)$ \\
10 & 0.0028 & 0.0259 & 0.0857 & 6.1959 & -0.2489 & 0.1066 & $0.9642(0.4797)$ \\
11 & 0.0029 & 0.0265 & 0.0792 & 6.1399 & -0.2489 & 0.1082 & $0.9790(0.6107)$ \\
12 & 0.0029 & 0.0263 & 0.1165 & 6.0007 & -0.2488 & 0.1093 & $0.9893(0.7099)$ \\
13 & 0.0029 & 0.0260 & 0.0742 & 5.9680 & -0.2488 & 0.1099 & $0.9942(0.7754)$ \\
14 & 0.0029 & 0.0261 & 0.0722 & 5.9641 & -0.2488 & 0.1101 & $0.9960(0.8268)$ \\
15 & 0.0029 & 0.0261 & 0.0727 & 5.8957 & -0.2488 & 0.1102 & $0.9974(0.8518)$ \\
16 & 0.0029 & 0.0262 & 0.0832 & 5.9889 & -0.2488 & 0.1105 & 1 \\
\hline
\end{tabular}

This table reports the in-sample mean $(\hat{\mu})$, standard deviation $(\hat{\sigma})$, skewness $(\hat{\xi})$, kurtosis $(\hat{\kappa})$, Expected Utility $(\hat{U})$ and the in-sample Sharpe ratio $(\hat{S})$ of each efficient MVS expected utility/cardinality portfolio. It also reports the ratio, $\hat{S} / \hat{S}_{16}$, between the in-sample Sharpe ratio of each efficient portfolio and the in-sample Sharpe ratio of the efficient portfolio with cardinality 16 (the highest Sharpe ratio portfolio). The Ledoit and Wolf (2008) bootstrap $p$-values for the statistical significance of the difference between the in-sample Sharpe ratio of the efficient portfolio with cardinality 16 and the respective efficient portfolio are presented in parenthesis. 
Table 6: In-sample analysis of the efficient MVSK expected utility/cardinality portfolios

\begin{tabular}{c|ccccccc}
\hline \multicolumn{10}{c}{} & \multicolumn{7}{c}{ Efficient portfolios } \\
\hline \hline Number of stocks & $\hat{\mu}$ & $\hat{\sigma}$ & $\hat{\xi}$ & $\hat{\kappa}$ & $\hat{U}$ & $\hat{S}$ & $\hat{S} / \hat{S}_{16}$ \\
\hline 1 & 0.0008 & 0.0204 & -0.3441 & 8.4074 & -0.2503 & 0.0385 & $0.3497(0.0038)$ \\
2 & 0.0006 & 0.0162 & -0.3841 & 7.5402 & -0.2500 & 0.0395 & $0.3589(0.0044)$ \\
3 & 0.0019 & 0.0232 & -0.1020 & 5.2611 & -0.2495 & 0.0817 & $0.7418(0.0566)$ \\
4 & 0.0019 & 0.0222 & -0.1086 & 5.3120 & -0.2494 & 0.0846 & $0.7675(0.0624)$ \\
5 & 0.0023 & 0.0245 & -0.0176 & 4.8963 & -0.2492 & 0.0928 & $0.8426(0.1488)$ \\
6 & 0.0024 & 0.0247 & 0.0047 & 5.0890 & -0.2491 & 0.0966 & $0.8763(0.1948)$ \\
7 & 0.0025 & 0.0246 & 0.1855 & 6.0367 & -0.2490 & 0.1005 & $0.9124(0.2460)$ \\
8 & 0.0025 & 0.0243 & 0.2066 & 6.4703 & -0.2490 & 0.1015 & $0.9209(0.2759)$ \\
9 & 0.0026 & 0.0249 & 0.1721 & 6.0183 & -0.2490 & 0.1033 & $0.9375(0.3251)$ \\
10 & 0.0027 & 0.0254 & 0.0649 & 6.0057 & -0.2489 & 0.1062 & $0.9638(0.4711)$ \\
11 & 0.0028 & 0.0259 & 0.0559 & 5.9411 & -0.2489 & 0.1078 & $0.9783(0.6153)$ \\
12 & 0.0028 & 0.0257 & 0.0920 & 5.7790 & -0.2489 & 0.1090 & $0.9891(0.7141)$ \\
13 & 0.0028 & 0.0255 & 0.0524 & 5.7804 & -0.2488 & 0.1096 & $0.9943(0.7774)$ \\
14 & 0.0028 & 0.0255 & 0.0517 & 5.7863 & -0.2488 & 0.1098 & $0.9960(0.8246)$ \\
15 & 0.0028 & 0.0255 & 0.0523 & 5.7182 & -0.2488 & 0.1099 & $0.9975(0.8472)$ \\
16 & 0.0028 & 0.0257 & 0.0599 & 5.7839 & -0.2488 & 0.1102 & 1 \\
\hline
\end{tabular}

This table reports the in-sample mean $(\hat{\mu})$, standard deviation $(\hat{\sigma})$, skewness $(\hat{\xi})$, kurtosis $(\hat{\kappa})$, Expected Utility $(\hat{U})$ and the in-sample Sharpe ratio $(\hat{S})$ of each efficient MVSK expected utility/cardinality portfolio. It also reports the ratio, $\hat{S} / \hat{S}_{16}$, between the in-sample Sharpe ratio of each efficient portfolio and the in-sample Sharpe ratio of the efficient portfolio with cardinality 16 (the highest Sharpe ratio portfolio). The Ledoit and Wolf (2008) bootstrap p-values for the statistical significance of the difference between the in-sample Sharpe ratio of the efficient portfolio with cardinality 16 and the respective efficient portfolio are presented in parenthesis.

One interesting result is that, in each of the three frameworks, the in-sample certainty equivalent and Sharpe ratio increases gradually with cardinality, until the maximum cardinality level of 16 is achieved. Moreover, we computed the bootstrap $p$-values for the statistical significance of the difference between the in-sample Sharpe ratio of the efficient portfolio with cardinality 16 and each other efficient portfolio. These $p$-values were computed according the Ledoit and Wolf (2008) robust methodology. For the MV framework these bootstrap $p$-values show that only the portfolios with cardinality 1, 2 and 3, consistently offer a Sharpe ratio significantly lower than the Sharpe ratio of the portfolio with cardinality 16. For the MVS and MVSK frameworks, this happens only for the portfolios with cardinality 1 and 2. This seems to suggest that, for this dataset, most of the diversification gains occur at cardinalities of 3 or 4 .

In these tables, it may seem puzzling that there is not a clear decreasing trend of the standard deviation of portfolio returns with the increase of cardinality. However, we must point out that we are, in fact, maximizing the expected utility for each level of cardinality. Therefore, with a marginal increase in the cardinality level, we may choose a completely different set of stocks (and associated weights) for which the benefits resulting from other moments of the return distribution (expected return and, when applicable, skewness and/or kurtosis) more than offset a possible increase in the standard deviation.

Results reported in Tables 3, 4, 5 and 6 point out that there are no significant differences 
between the three frameworks in terms of in-sample certainty equivalent and Sharpe ratio. For example, in each of the three frameworks the maximum certainty equivalent return is achieved at the maximum cardinality level and the values of $\widehat{C E}$ are quite similar $(0.11956 \%, 0.11989 \%$ and $0.11760 \%$, respectively). Also the maximum in-sample Sharpe ratio is found, in each of the three frameworks, at the maximum feasible cardinality level and with similar values for $\hat{S}(11.06 \%$, $11.05 \%$ and $11.02 \%$, respectively). These results suggest that, in-sample, the consideration of higher moments does not produce relevant gains (in terms of certainty equivalent) for an investor with CRRA preferences $(\gamma=5)$.

\subsection{Out-of-sample performance}

The out-of-sample analysis relies on a rolling-sample approach, with an estimation window of 1565 days. The initial estimation window begins in July 2007 and ends in June 2013. There are 261 evaluation periods (days) until June 2014.

In Table 7 it is reported the out-of-sample certainty equivalent return (computed according Equation (10)), $\widehat{C E}$, for each one of the three frameworks.

Table 7: Out-of-sample certainty equivalent return

\begin{tabular}{c|r|r|r}
\hline \multicolumn{4}{c}{ Efficient portfolios } \\
\hline \hline Number of stocks & MV & MVS & MVSK \\
\hline 1 & -0.3605 & -0.1337 & -0.2635 \\
2 & -0.2165 & -0.2886 & -0.5271 \\
3 & -1.3200 & -1.2945 & -1.1657 \\
4 & -1.4815 & -1.4850 & -1.6058 \\
5 & -0.5165 & -0.7985 & -1.1355 \\
6 & -1.2108 & -1.0303 & -1.0729 \\
7 & -0.9808 & -1.3350 & -1.0559 \\
8 & -1.5770 & -1.2233 & -1.2436 \\
9 & -1.3603 & -1.0098 & -1.1505 \\
10 & -0.7319 & -1.0514 & -0.7085 \\
11 & -0.8519 & -0.9998 & -0.9240 \\
12 & -0.8622 & -0.8066 & -0.5946 \\
13 & -0.8368 & -0.7737 & -0.6427 \\
14 & -0.7461 & -0.7586 & -0.5603 \\
15 & -0.7741 & -0.7319 & -0.6841 \\
16 & -0.7593 & -0.7701 & -0.7048 \\
\hline
\end{tabular}

This table lists the out-of-sample certainty equivalent return $\widehat{(\widehat{C E})}$ of the efficient expected utility/cardinality portfolios. $M V$ refers to the efficient $M V$ expected utility/cardinality portfolios, MVS refers to the efficient MVS expected utility/cardinality portfolios and MVSK refers to the efficient MVSK expected utility/cardinality portfolios. All the certainty equivalent values are multiplied by a factor of 1000 .

Tables 8, 9 and 10 report some out-of-sample statistics (the out-of-sample mean, $\hat{\mu}$, standard deviation, $\hat{\sigma}$, skewness, $\hat{\xi}$, kurtosis, $\hat{\kappa}$, expected utility, $\hat{U}$, and Sharpe ratio, $\hat{S}$ ) for the MV, MVS and MVSK frameworks, respectively. When the numerator of the Sharpe ratio $(\hat{S})$ is negative, 
it should be refined in order to achieve a correct rank of the portfolios. The most widely used methodology to refine the Sharpe ratio is the Israelsen (2005) methodology:

$$
\hat{S}_{r e f}=\frac{\hat{\mu}}{\hat{\sigma}^{\hat{\mu} / a b s(\hat{\mu})}},
$$

where $\operatorname{abs}($.$) is the absolute value function. Note that the refined Sharpe ratio \left(\hat{S}_{\text {ref }}\right)$ is equal to the Sharpe ratio $(\hat{S})$ when the numerator is non-negative; in this case, Equation (11) is equivalent to Equation (12).

Table 8: Out-of-sample analysis of the efficient MV expected utility/cardinality portfolios

\begin{tabular}{|c|c|c|c|c|c|c|c|c|}
\hline \multicolumn{9}{|c|}{ Efficient portfolios } \\
\hline Number of stocks & $\hat{\mu}$ & $\hat{\sigma}$ & $\hat{\xi}$ & $\hat{\kappa}$ & $\hat{U}$ & $\hat{S}$ & $\hat{S} / \hat{S}_{16}$ & $\hat{S}_{r e f}$ \\
\hline 1 & 0.0001 & 0.0137 & -0.1952 & 4.1943 & -0.2504 & 0.0084 & $0.1472(0.5781)$ & 0.0084 \\
\hline 2 & 0.0002 & 0.0137 & 0.8597 & 8.7178 & -0.2502 & 0.0177 & $0.3105(0.5961)$ & 0.0177 \\
\hline 3 & -0.0002 & 0.0209 & -0.3217 & 5.2709 & -0.2513 & -0.0100 & $-0.1750(0.0888)$ & -0.0000 \\
\hline 4 & -0.0001 & 0.0231 & 0.0797 & 4.9952 & -0.2515 & -0.0065 & $-0.1137(0.0624)$ & -0.0000 \\
\hline 5 & 0.0011 & 0.0249 & -0.3433 & 4.3266 & -0.2505 & 0.0430 & $0.7555(0.6273)$ & 0.0430 \\
\hline 6 & 0.0006 & 0.0266 & -0.4120 & 5.1481 & -0.2512 & 0.0231 & $0.4060(0.1562)$ & 0.0231 \\
\hline 7 & 0.0009 & 0.0272 & -0.2345 & 3.8378 & -0.2510 & 0.0329 & $0.5784(0.1998)$ & 0.0329 \\
\hline 8 & 0.0004 & 0.0278 & -0.3922 & 4.6299 & -0.2516 & 0.0146 & $0.2569(0.0282)$ & 0.0146 \\
\hline 9 & 0.0008 & 0.0287 & -0.3905 & 4.4395 & -0.2514 & 0.0266 & $0.4675(0.0750)$ & 0.0266 \\
\hline 10 & 0.0015 & 0.0294 & -0.3697 & 4.5972 & -0.2507 & 0.0508 & $0.8928(0.6575)$ & 0.0508 \\
\hline 11 & 0.0015 & 0.0301 & -0.3385 & 4.3533 & -0.2509 & 0.0490 & $0.8606(0.4619)$ & 0.0490 \\
\hline 12 & 0.0016 & 0.0309 & -0.4063 & 4.5166 & -0.2509 & 0.0520 & $0.9129(0.8708)$ & 0.0520 \\
\hline 13 & 0.0017 & 0.0312 & -0.3999 & 4.5208 & -0.2508 & 0.0538 & $0.9456(0.5527)$ & 0.0538 \\
\hline 14 & 0.0018 & 0.0314 & -0.3855 & 4.6060 & -0.2507 & 0.0573 & $1.0064(0.9100)$ & 0.0573 \\
\hline 15 & 0.0018 & 0.0314 & -0.3909 & 4.5925 & -0.2508 & 0.0563 & $0.9885(0.6667)$ & 0.0563 \\
\hline 16 & 0.0018 & 0.0314 & -0.4056 & 4.6114 & -0.2508 & 0.0569 & 1 & 0.0569 \\
\hline
\end{tabular}

This table reports the out-of-sample mean $(\hat{\mu})$, standard deviation $(\hat{\sigma})$, skewness $(\hat{\xi})$, kurtosis $(\hat{\kappa})$, Expected Utility $(\hat{U})$ and the out-of-sample Sharpe ratio $(\hat{S})$ of each efficient $M V$ expected utility/cardinality portfolio. It also reports the ratio, $\hat{S} / \hat{S}_{16}$, between the out-of-sample Sharpe ratio of each efficient portfolio and the out-ofsample Sharpe ratio of the efficient portfolio with cardinality 16. The Ledoit and Wolf (2008) bootstrap p-values for the statistical significance of the difference between the in-sample Sharpe ratio of the efficient portfolio with cardinality 16 and the respective efficient portfolio are presented in parenthesis. The out-of-sample refined Sharpe ratio $\left(\hat{S}_{\text {ref }}\right.$ ) of each efficient $M V$ expected utility/cardinality portfolio is also reported. The refined out-of-sample Sharpe ratio was computed according to the Israelsen (2005) methodology. 
Table 9: Out-of-sample analysis of the efficient MVS expected utility/cardinality portfolios

\begin{tabular}{|c|c|c|c|c|c|c|c|c|}
\hline \multicolumn{9}{|c|}{ Efficient portfolios } \\
\hline Number of stocks & $\hat{\mu}$ & $\overline{\hat{\sigma}}$ & $\hat{\hat{\xi}}$ & $\hat{\kappa}$ & $\hat{\hat{U}}$ & $\hat{S}$ & $\hat{S} / \hat{S}_{16}$ & $\hat{S}_{r e f}$ \\
\hline 1 & 0.0003 & 0.0137 & -0.2027 & 4.2231 & -0.2501 & 0.0249 & $0.4410(0.7243)$ & 0.0249 \\
\hline 2 & 0.0002 & 0.0134 & 0.8635 & 9.2178 & -0.2503 & 0.0113 & $0.2001(0.5447)$ & 0.0113 \\
\hline 3 & -0.0002 & 0.0208 & -0.3166 & 5.3093 & -0.2513 & -0.0091 & $-0.1618(0.1044)$ & -0.0000 \\
\hline 4 & -0.0001 & 0.0231 & -0.0339 & 5.2009 & -0.2515 & -0.0059 & $-0.1053(0.0726)$ & -0.0000 \\
\hline 5 & 0.0008 & 0.0254 & -0.3214 & 4.1134 & -0.2508 & 0.0334 & $0.5918(0.3915)$ & 0.0334 \\
\hline 6 & 0.0007 & 0.0264 & -0.2889 & 4.2218 & -0.2510 & 0.0282 & $0.4994(0.2456)$ & 0.0282 \\
\hline 7 & 0.0006 & 0.0274 & -0.4334 & 4.5700 & -0.2513 & 0.0219 & $0.3888(0.0690)$ & 0.0219 \\
\hline 8 & 0.0007 & 0.0278 & -0.3456 & 4.1169 & -0.2512 & 0.0270 & $0.4788(0.0852)$ & 0.0270 \\
\hline 9 & 0.0011 & 0.0287 & -0.3050 & 4.4441 & -0.2510 & 0.0382 & $0.6770(0.2444)$ & 0.0382 \\
\hline 10 & 0.0011 & 0.0290 & -0.3803 & 4.5557 & -0.2511 & 0.0385 & $0.6829(0.1652)$ & 0.0385 \\
\hline 11 & 0.0013 & 0.0302 & -0.3448 & 4.4666 & -0.2510 & 0.0445 & $0.7889(0.3589)$ & 0.0445 \\
\hline 12 & 0.0016 & 0.0306 & -0.3728 & 4.5447 & -0.2508 & 0.0523 & $0.9269(0.9046)$ & 0.0523 \\
\hline 13 & 0.0017 & 0.0311 & -0.4084 & 4.5232 & -0.2508 & 0.0555 & $0.9840(0.8580)$ & 0.0555 \\
\hline 14 & 0.0018 & 0.0313 & -0.3878 & 4.5733 & -0.2508 & 0.0565 & $1.0026(0.9690)$ & 0.0565 \\
\hline 15 & 0.0018 & 0.0313 & -0.3999 & 4.5931 & -0.2507 & 0.0575 & $1.0196(0.5287)$ & 0.0575 \\
\hline 16 & 0.0018 & 0.0313 & -0.4143 & 4.5854 & -0.2508 & 0.0564 & 1 & 0.0564 \\
\hline
\end{tabular}

This table reports the out-of-sample mean $(\hat{\mu})$, standard deviation $(\hat{\sigma})$, skewness $(\hat{\xi})$, kurtosis $(\hat{\kappa})$, Expected Utility $(\hat{U})$ and the out-of-sample Sharpe ratio $(\hat{S})$ of each efficient MVS expected utility/cardinality portfolio. It also reports the ratio, $\hat{S} / \hat{S}_{16}$, between the out-of-sample Sharpe ratio of each efficient portfolio and the out-ofsample Sharpe ratio of the efficient portfolio with cardinality 16. The Ledoit and Wolf (2008) bootstrap p-values for the statistical significance of the difference between the in-sample Sharpe ratio of the efficient portfolio with cardinality 16 and the respective efficient portfolio are presented in parenthesis. The out-of-sample refined Sharpe ratio $\left(\hat{S}_{r e f}\right.$ ) of each efficient MVS expected utility/cardinality portfolio is also reported. The refined out-of-sample Sharpe ratio was computed according to the Israelsen (2005) methodology. 
Table 10: Out-of-sample analysis of the efficient MVSK expected utility/cardinality portfolios

\begin{tabular}{|c|c|c|c|c|c|c|c|c|}
\hline \multicolumn{9}{|c|}{ Efficient portfolios } \\
\hline Number of stocks & $\hat{\mu}$ & $\overline{\hat{\sigma}}$ & $\hat{\hat{\xi}}$ & $\hat{\kappa}$ & $\hat{U}$ & $\hat{S}$ & $\hat{S} / \hat{S}_{16}$ & $\hat{S}_{r e f}$ \\
\hline 1 & 0.0002 & 0.0138 & -0.1811 & 4.1731 & -0.2503 & 0.0155 & $0.2763(0.6363)$ & 0.0155 \\
\hline 2 & -0.0001 & 0.0123 & -0.2762 & 4.3074 & -0.2505 & -0.0116 & $-0.2069(0.3833)$ & -0.0000 \\
\hline 3 & -0.0001 & 0.0206 & -0.3015 & 5.5200 & -0.2512 & -0.0042 & $-0.0752(0.1414)$ & -0.0000 \\
\hline 4 & -0.0003 & 0.0228 & 0.0790 & 4.9650 & -0.2516 & -0.0130 & $-0.2318(0.0508)$ & -0.0000 \\
\hline 5 & 0.0005 & 0.0251 & -0.2708 & 4.3071 & -0.2511 & 0.0185 & $0.3304(0.1702)$ & 0.0185 \\
\hline 6 & 0.0006 & 0.0257 & -0.2989 & 4.3997 & -0.2511 & 0.0238 & $0.4246(0.1846)$ & 0.0238 \\
\hline 7 & 0.0007 & 0.0265 & -0.2967 & 3.8895 & -0.2511 & 0.0277 & $0.4939(0.1394)$ & 0.0277 \\
\hline 8 & 0.0007 & 0.0273 & -0.4212 & 4.4027 & -0.2512 & 0.0248 & $0.4410(0.1260)$ & 0.0248 \\
\hline 9 & 0.0008 & 0.0274 & -0.4249 & 4.5070 & -0.2512 & 0.0283 & $0.5044(0.0764)$ & 0.0283 \\
\hline 10 & 0.0014 & 0.0283 & -0.4169 & 4.5242 & -0.2507 & 0.0480 & $0.8545(0.4993)$ & 0.0480 \\
\hline 11 & 0.0013 & 0.0295 & -0.4497 & 4.6709 & -0.2509 & 0.0448 & $0.7974(0.3523)$ & 0.0448 \\
\hline 12 & 0.0017 & 0.0298 & -0.3885 & 4.6517 & -0.2506 & 0.0567 & $1.0100(0.9812)$ & 0.0567 \\
\hline 13 & 0.0017 & 0.0304 & -0.3968 & 4.5750 & -0.2506 & 0.0573 & $1.0199(0.8254)$ & 0.0573 \\
\hline 14 & 0.0019 & 0.0307 & -0.3844 & 4.6244 & -0.2506 & 0.0608 & $1.0838(0.1760)$ & 0.0608 \\
\hline 15 & 0.0017 & 0.0307 & -0.4052 & 4.6198 & -0.2507 & 0.0570 & $1.0148(0.6133)$ & 0.0570 \\
\hline 16 & 0.0017 & 0.0307 & -0.4072 & 4.6057 & -0.2507 & 0.0561 & 1 & 0.0561 \\
\hline
\end{tabular}

This table reports the out-of-sample mean $(\hat{\mu})$, standard deviation $(\hat{\sigma})$, skewness $(\hat{\xi})$, kurtosis $(\hat{\kappa})$, Expected Utility $(\hat{U})$ and the out-of-sample Sharpe ratio $(\hat{S})$ of each efficient MVSK expected utility/cardinality portfolio. It also reports the ratio, $\hat{S} / \hat{S}_{16}$, between the out-of-sample Sharpe ratio of each efficient portfolio and the out-of-sample Sharpe ratio of the efficient portfolio with cardinality 16. The Ledoit and Wolf (2008) bootstrap p-values for the statistical significance of the difference between the in-sample Sharpe ratio of the efficient portfolio with cardinality 16 and the respective efficient portfolio are presented in parenthesis. The out-of-sample refined Sharpe ratio $\left(\hat{S}_{\text {ref }}\right)$ of each efficient MVSK expected utility/cardinality portfolio is also reported. The refined out-of-sample Sharpe ratio was computed according to the Israelsen (2005) methodology.

The out-of-sample certainty equivalent does not exhibit a clear trend with cardinality. In the MV framework, the best out-of-sample certainty equivalent is achieved for the portfolio with cardinality equal to $2(-0.02165 \%)$. The bootstrap $p$-values (computed according the classical methodology of Efron and Tibshirani, 1994) for the difference between the out-of-sample certainty equivalent of the efficient MV portfolio with cardinality 2 and the out-of-sample certainty equivalent of the MVS and MVSK portfolios with the same cardinality level (equal to 2) are both equal to 0.000. However, in the MVS and MVSK frameworks, the best out-of-sample certainty equivalent return is achieved for the portfolios with cardinality equal to $1(-0.01337 \%$ and $-0.02635 \%$, respectively for each framework). The bootstrap $p$-values for the difference between the out-of-sample certainty equivalent of the efficient MV portfolio with cardinality 1 and the out-of-sample certainty equivalent of the MVS and MVSK portfolios with the same cardinality level (equal to 1) are both equal to 0.000. These results suggest gains in considering skewness and kurtosis, for some cardinality levels. As we can see in Table 7, both the efficient MVS and the efficient MVSK portfolios outperform the efficient MV portfolios for eight cardinality levels (cardinality equal to $1,3,6,8,9,12,13$ and 15 ).

From the analysis of the refined Sharpe ratios, we conclude that the best out-of-sample performance is achieved by portfolios with cardinality 14, for the MV and MVSK frameworks, 
and 15, for the MVS framework. For these cardinalities, the best portfolio is either the one obtained within the MVSK framework (for cardinality 14) or the one obtained within the MVS framework (for cardinality 15). However, we cannot reach strong conclusions regarding whether or not there are gains in considering higher moments, since the bootstrap $p$-values indicate that the performance of these portfolios is never significantly better than the one of the portfolios obtained within the MV framework.

Tables 8 to 10 also report, for each of the three frameworks, the ratio, $\hat{S} / \hat{S}_{16}$, between the out-of-sample Sharpe ratio of each efficient portfolio and the out-of-sample Sharpe ratio of the efficient portfolio corresponding to the maximum cardinality of 16 . Based on these ratios, we computed (according to the methodology of Ledoit and Wolf 2008) the bootstrap $p$-values for the statistical significance of the difference between the out-of-sample Sharpe ratio of the efficient portfolio with cardinality 16 and each other efficient portfolio. Except for the difference between the out-of-sample Sharpe ratio of the MV portfolio with cardinality 8 and the MV portfolio with cardinality 16 (where the first is lower than the second), none of these differences were significant. This means that, for the three frameworks and except to the previous referred case, none of the efficient expected utility/cardinality portfolios presents a Sharpe ratio significantly higher or significantly lower than the Sharpe ratio of the efficient portfolio with cardinality 16.

Table 11 reports the bootstrap $p$-values of the difference between the out-of-sample Sharpe ratio of each pair of frameworks (MV vs MVS, MV vs MVSK, and MVS vs MVSK) for the same cardinality level. The efficient MVS portfolios outperform the MV portfolios, in terms of Sharpe ratio, for nine cardinality levels $(1,3,4,6,8,9,12,13$ and 15). Also for nine cardinality levels $(1,3,6,8,9,12,13,14,15)$, the efficient MVSK portfolios outperform the MV portfolios. In total, for $75 \%$ of the cardinality levels (12 in 16), the efficient MV portfolios are outperformed either by the corresponding efficient MVS portfolios or by the corresponding efficient MVSK portfolios (although none of the differences are statistically significant at the $5 \%$ significance level). This suggests that, for those cardinality levels, there may be small gains in considering higher moments (skewness and kurtosis). 
Table 11: Bootstrap p-values for the Sharpe ratios

\begin{tabular}{|c|c|c|c|}
\hline \multicolumn{4}{|c|}{ Efficient portfolios } \\
\hline Number of stocks & MV vs MVS & MV vs MVSK & MVS vs MVSK \\
\hline 1 & 0.1794 (MVS) & 0.4851 (MVSK) & 0.1648 (MVS) \\
\hline 2 & $0.5515(\mathrm{MV})$ & $0.4803(\mathrm{MV})$ & 0.4713 (MVS) \\
\hline 3 & 0.8930 (MVS) & 0.3801 (MVSK) & 0.3439 (MVSK) \\
\hline 4 & 0.9540 (MVS) & 0.5007 (MV) & 0.3787 (MVS) \\
\hline 5 & $0.4251(\mathrm{MV})$ & $0.0658(\mathrm{MV})$ & 0.1792 (MVS) \\
\hline 6 & 0.6323 (MVS) & 0.9478 (MVSK) & 0.6743 (MVS) \\
\hline 7 & $0.1862(\mathrm{MV})$ & $0.6331(\mathrm{MV})$ & 0.5469 (MVSK) \\
\hline 8 & 0.4759 (MVS) & 0.5353 (MVSK) & 0.8630 (MVS) \\
\hline 9 & 0.4171 (MVS) & 0.8924 (MVSK) & 0.2198 (MVS) \\
\hline 10 & 0.1746 (MV) & 0.7409 (MV) & 0.2513 (MVSK) \\
\hline 11 & 0.4935 (MV) & $0.5563(\mathrm{MV})$ & 0.9672 (MVSK) \\
\hline 12 & 0.9658 (MVS) & 0.4541 (MVSK) & 0.3415 (MVSK) \\
\hline 13 & 0.7003 (MVS) & 0.3705 (MVSK) & 0.2941 (MVSK) \\
\hline 14 & 0.7960 (MV) & 0.1132 (MVSK) & 0.0426 (MVSK) \\
\hline 15 & 0.6179 (MVS) & 0.7844 (MVSK) & 0.7674 (MVS) \\
\hline 16 & 0.7816 (MV) & 0.6415 (MV) & 0.7890 (MVS) \\
\hline
\end{tabular}

This table lists the bootstrap p-values of the difference between the Sharpe ratio of each pair of frameworks. $M V$ refers to the efficient $M V$ expected utility/cardinality portfolios, MVS refers to the efficient MVS expected utility/cardinality portfolios, and MVSK refers to the efficient MVSK expected utility/cardinality portfolios. The bootstrap p-values were computed according the Ledoit and Wolf (2008) methodology. In parenthesis there is the indication of the framework with the highest Sharpe ratio.

Given the time series of daily out-of-sample returns, for each efficient portfolio considered, we computed the portfolio turnover, defined as the average, over all time periods, of the absolute changes in weights (corresponding to trades) across the $N$ available stocks:

$$
\text { turnover }=\frac{1}{\# \text { periods }} \sum_{t=1}^{\# \text { periods }} \sum_{i=1}^{N}\left(\left|w_{i, t+1}-w_{i, t}^{h}\right|\right),
$$

where $w_{i, t+1}$ is the portfolio weight after rebalancing at time $t+1$ and $w_{i, t}^{h}$ is the portfolio weight before rebalancing at time $t+1$. Thus, $w_{i, t}^{h}$, is computed as

$$
w_{i, t}^{h}=w_{i, t-1} \frac{1+r_{i, t}}{1+r_{p, t}},
$$

where $r_{i, t}$ is the return at time $t$ of the stock $i$ and $r_{p, t}$ is the return at time $t$ of the portfolio. The results are reported in Table 12. In all the three frameworks, the turnover begins by increasing up to a certain cardinality level (around 8 or 9 , which corresponds to about $50 \%$ of the maximum cardinality level). This is somehow expected, since the increase in the number of stocks should increase the transaction costs. However, after reaching that cardinality level, we observe, in all the three frameworks, the non-intuitive fact that the turnover decreases when the cardinality increases. The explanation for this result is that the small fluctuations of the portfolio weights 
compensate the increment of the number of stocks in the portfolio. Thus, in this case, from the cardinality level of about 8 (around $50 \%$ of the maximum cardinality), increasing the cardinality of a portfolio allows the investor to reduce transaction costs.

Table 12: Portfolio turnover

\begin{tabular}{c|r|r|r}
\hline \multicolumn{4}{c}{ Efficient portfolios } \\
\hline \hline Number of stocks & MV & MVS & MVSK \\
\hline 1 & 5.25 & 3.77 & 5.85 \\
2 & 12.12 & 9.77 & 12.99 \\
3 & 11.28 & 11.63 & 12.76 \\
4 & 45.33 & 38.22 & 44.93 \\
5 & 44.76 & 45.26 & 60.58 \\
6 & 55.28 & 50.59 & 60.77 \\
7 & 53.20 & 49.32 & 59.98 \\
8 & 69.72 & 60.92 & 78.71 \\
9 & 61.65 & 62.82 & 76.84 \\
10 & 47.14 & 51.18 & 56.61 \\
11 & 44.43 & 47.87 & 53.42 \\
12 & 32.58 & 39.67 & 43.48 \\
13 & 33.51 & 32.57 & 34.44 \\
14 & 33.66 & 33.24 & 36.80 \\
15 & 30.10 & 29.10 & 33.17 \\
16 & 28.98 & 29.76 & 33.43 \\
\hline
\end{tabular}

This table lists the portfolio turnover of the efficient expected utility/cardinality portfolios. MV refers to the efficient MV expected utility/cardinality portfolios, MVS refers to the efficient MVS expected utility/cardinality portfolios and MVSK refers to the efficient MVSK expected utility/cardinality portfolios.

In order to assess the impact of transaction costs in the performance of the different strategies, we computed the out-of-sample Sharpe ratio of returns net of transaction costs, defined as

$$
\hat{S}_{t c}=\frac{\hat{\mu}_{t c}}{\hat{\sigma}_{t c}}
$$

where $\hat{\mu}_{t c}=\hat{\mu}-t c$ is the out-of-sample time series of the mean of the returns $(\hat{\mu})$ deducted by transaction costs $(t c)$ and $\hat{\sigma}_{t c}$ is the standard deviation of the out-of-sample returns after transaction costs. We used proportional transaction costs defined as ${ }^{6}$

$$
t c=\sum_{t=1}^{\# \text { periods }} 0.3 \% \sum_{i=1}^{N}\left(\left|w_{i, t+1}-w_{i, t}^{h}\right|\right) .
$$

The refined Sharpe ratios after transaction costs for the efficient portfolios are reported in Table 13. The inclusion of transaction costs may lead to completely different results (see, e.g., Brito

\footnotetext{
${ }^{6}$ According to the information received from several stock brokers in the Lisbon Stock Exchange (Euronext Lisbon), for the stocks in the PSI20 index the proportional transaction cost was set equal to 30 basis points per transaction.
} 
and Vicente, 2014; DeMiguel et al., 2014; DeMiguel and Olivares-Nadal, 2015). Not taking into account the transaction costs in the out-of-sample analysis may result in the misleading conclusion that diversification is not an important issue in portfolio management. For example, Brodie et al. (2009) have implemented a specific algorithm in order to construct sparse portfolios (portfolios with low cardinality) in the classical mean-variance setting. Their empirical out-of-sample results, for two sets of portfolios constructed by Fama and French (FF48 and FF100), indicate that sparse portfolios outperform (in terms of Sharpe ratio) the equally weighted portfolio, suggesting that diversification does not produce positive results out-of-sample (for the FF48 dataset, the Sharpe ratio even exhibits a decreasing trend with cardinality). However, the authors do not account for transaction costs, limiting the real applicability of their findings.

The results that we obtained for the net Sharpe ratios highlight that in the three frameworks, the pattern of the net Sharpe ratios is inversely related with the turnover pattern, i.e., the net Sharpe ratios decrease to a certain level of cardinality (around 8 or 9) and then increases with the cardinality level. For all frameworks, the portfolio with the minimum cardinality of 1 is the portfolio with the highest refined Sharpe ratio after transaction costs. This is in agreement with previous studies suggesting that sparse portfolios tend to exhibit a good performance (see, e.g., Brodie et al., 2009). However, the results that we obtain go further and suggest that for sufficiently large levels of cardinality, there are gains with diversification.

Table 13: Refined Sharpe ratios of returns net of transaction costs

\begin{tabular}{c|c|c|c}
\hline \multicolumn{4}{c}{ Efficient portfolios } \\
\hline \hline Number of stocks & MV & MVS & MVSK \\
\hline 1 & -0.0566 & -0.0406 & -0.0631 \\
2 & -0.1297 & -0.1024 & -0.1255 \\
3 & -0.1845 & -0.1896 & -0.2058 \\
4 & -0.8184 & -0.6921 & -0.8038 \\
5 & -0.8744 & -0.9002 & -1.1893 \\
6 & -1.1531 & -1.0444 & -1.2229 \\
7 & -1.1312 & -1.0588 & -1.2446 \\
8 & -1.5160 & -1.3237 & -1.6839 \\
9 & -1.3875 & -1.4105 & -1.6457 \\
10 & -1.0867 & -1.1639 & -1.2563 \\
11 & -1.0476 & -1.1319 & -1.2325 \\
12 & -0.7891 & -0.9492 & -1.0138 \\
13 & -0.8198 & -0.7939 & -0.8202 \\
14 & -0.8280 & -0.8151 & -0.8845 \\
15 & -0.7394 & -0.7138 & -0.7976 \\
16 & -0.7126 & -0.7303 & -0.8026 \\
\hline
\end{tabular}

This table reports the refined Sharpe ratios of returns net of transaction costs for each efficient expected utility/cardinality portfolio. MV refers to the efficient $M V$ expected utility/cardinality portfolios, MVS refers to the efficient MVS expected utility/cardinality portfolios and MVSK refers to the efficient MVSK expected utility/cardinality portfolios. The refined net Sharpe ratios were computed according the Israelsen (2005) methodology. 
In Table 14 we report the bootstrap $p$-values of the difference between the Sharpe ratio after transaction costs for each pair of frameworks (MV vs MVS, MV vs MVSK, and MVS vs MVSK) given a specific cardinality level. From the comparison between the MV and the MVS portfolios we found that the MVS portfolios outperform the MV portfolios (the differences being statistically significant) for nine cardinality levels $(1,2,4,6,7,8,13,14$ and 15). Looking to the MV and the MVSK portfolios, we found that the MVSK portfolios outperform the MV portfolios only for two cardinality levels (2 and 4 ), but the differences are not statistically significant. Summarizing, the MV portfolios underperform at least one of the efficient portfolios from the two other frameworks for 9 of 16 cardinality levels. This indicates that when transaction costs are taken into account, there are gains (in terms of Sharpe ratio) in considering the skewness, for certain cardinality levels.

Table 14: Bootstrap $p$-values for the net Sharpe ratios

\begin{tabular}{c|l|l|l}
\hline \multicolumn{5}{c}{ Efficient portfolios } \\
\hline \hline Number of stocks & MV vs MVS & MV vs MVSK & MVS vs MVSK \\
\hline 1 & $0.0002(\mathrm{MVS})$ & $0.0002(\mathrm{MV})$ & $0.0002(\mathrm{MVS})$ \\
2 & $0.0002(\mathrm{MVS})$ & $0.1527(\mathrm{MVSK})$ & $0.0018(\mathrm{MVS})$ \\
3 & $0.0002(\mathrm{MV})$ & $0.0002(\mathrm{MV})$ & $0.0002(\mathrm{MVS})$ \\
4 & $0.0002(\mathrm{MVS})$ & $0.9999(\mathrm{MVSK})$ & $0.0002(\mathrm{MVS})$ \\
5 & 0.5037 (MV) & $0.0002(\mathrm{MV})$ & $0.0002(\mathrm{MVS})$ \\
6 & $0.0006(\mathrm{MVS})$ & $0.0002(\mathrm{MV})$ & $0.0002(\mathrm{MVS})$ \\
7 & $0.0002(\mathrm{MVS})$ & $0.0002(\mathrm{MV})$ & $0.0002(\mathrm{MVS})$ \\
8 & $0.0006(\mathrm{MVS})$ & $0.0002(\mathrm{MV})$ & $0.0002(\mathrm{MVS})$ \\
9 & $0.2841(\mathrm{MV})$ & $0.0002(\mathrm{MV})$ & $0.0002(\mathrm{MVS})$ \\
10 & $0.0002(\mathrm{MV})$ & $0.0002(\mathrm{MV})$ & $0.0002(\mathrm{MVS})$ \\
11 & $0.0002(\mathrm{MV})$ & $0.0002(\mathrm{MV})$ & $0.0002(\mathrm{MVS})$ \\
12 & $0.0002(\mathrm{MV})$ & $0.0002(\mathrm{MV})$ & $0.0002(\mathrm{MVS})$ \\
13 & $0.0002(\mathrm{MVS})$ & $0.0002(\mathrm{MV})$ & $0.0002(\mathrm{MVS})$ \\
14 & $0.0014(\mathrm{MVS})$ & $0.0002(\mathrm{MV})$ & $0.0002(\mathrm{MVS})$ \\
15 & $0.0002(\mathrm{MVS})$ & $0.0002(\mathrm{MV})$ & $0.0002(\mathrm{MVS})$ \\
16 & $0.0002(\mathrm{MV})$ & $0.0002(\mathrm{MV})$ & $0.0002(\mathrm{MVS})$ \\
\hline
\end{tabular}

This table lists the bootstrap p-values of the difference between the net Sharpe ratios of two different frameworks. $M V$ refers to the efficient $M V$ expected utility/cardinality portfolios, MVS refers to the efficient MVS expected utility/cardinality portfolios, and MVSK refers to the efficient MVSK expected utility/cardinality portfolios. The bootstrap p-values were computed according the Ledoit and Wolf (2008) methodology. The framework with the highest Sharpe ratio is indicated in parenthesis.

The cardinality becomes even a more important issue in datasets with a larger number of stocks. In order to provide an indication of whether certain patterns found in the results discussed above (for the PSI20 index) remain valid in larger datasets, we decided to present some results for a dataset from the EUROSTOXX50 index (see Table 15). 
Table 15: The EUROSTOXX50 dataset

\begin{tabular}{l|l}
\hline \multicolumn{2}{c}{ List of stocks } \\
\hline AIR LIQUIDE & IBERDROLA \\
AIRBUS GROUP & INDITEX \\
ALLIANZ (XET) & ING GROEP \\
ANHEUSER-BUSCH INBEV & INTESA SANPAOLO \\
ASML HOLDING & L'OREAL \\
ASSICURAZIONI GENERALI & LVMH \\
AXA & MUENCHENER RUCK (XET) \\
BANCO SANTANDER & ORANGE \\
BASF (XET) & PHILIPS ELTN KONINKLIJKE \\
BAYER (XET) & REPSOL YPF \\
BBV.ARGENTARIA & RWE (XET) \\
BMW (XET) & SAINT GOBAIN \\
BNP PARIBAS & SANOFI \\
CARREFOUR & SAP (XET) \\
CRH (DUB) & SCHNEIDER ELECTRIC SE \\
DAIMLER (XET) & SIEMENS (XET) \\
DANONE & SOCIETE GENERALE \\
DEUTSCHE BANK (XET) & TELEFONICA \\
DEUTSCHE POST (XET) & TOTAL \\
DEUTSCHE TELEKOM (XET) & UNIBAIL-RODAMCO \\
E ON (XET) & UNICREDIT \\
ENEL & UNILEVER CERTS \\
ENI & VINCI \\
ESSILOR INTL & VIVENDI \\
GDF SUEZ & VOLKSWAGEN PREF (XET) \\
\hline
\end{tabular}

This table lists the composition of the EUROSTOXX50 dataset. Daily closing prices, from July 2007 to June 2014, of these 50 stocks (from the EUROSTOXX50 index) were collected from the Thomson Reuters Datastream ${ }^{\circledR}$.

Similarly to the PSI20 dataset, the daily discrete returns of the EUROSTOXX50 dataset (see Table 16), presented, on average, negative but near zero mean, positive skewness and above normal kurtosis. 
Table 16: Descriptive statistics for the EUROSTOXX50 dataset

\begin{tabular}{l|l}
\hline \hline Number of days & 1826 \\
\hline Minimum & -0.12984 \\
\hline Median & 0.00006 \\
\hline Maximum & 0.16665 \\
\hline Mean & 0.00019 \\
\hline Variance & 0.00051 \\
\hline Skewness & 0.33992 \\
\hline Kurtosis & 10.2218 \\
\hline
\end{tabular}

This table reports some descriptive statistics for the EUROSTOXX50 dataset (the composition of the dataset is reported in Table 15). These statistics are averaged cross-sectionally, i.e., they are computed for each stock and then the arithmetic mean is taken.

We applied, to the EUROSTOXX50 dataset, exactly the same rolling-sample approach described in the beginning of this Section. Table 17 reports the out-of-sample certainty equivalent (solution of Equation 10), $\widehat{C E}$, for the MV, MVS and MVSK frameworks ${ }^{7}$.

\footnotetext{
${ }^{7}$ Similarly to the PSI20 dataset case, the introduction of a threshold of $1 \%$ for the cardinality computation reduced the maximum cardinality level that led to non-dominated solutions. In the case of the EUROSTOXX50 dataset the maximum cardinality is equal to 40 .
} 
Table 17: Certainty equivalent return for the EUROSTOXX50 dataset

\begin{tabular}{c|r|r|r}
\hline \multicolumn{4}{r}{ Efficient portfolios } \\
\hline \hline Number of stocks & MV & MVS & MVSK \\
\hline 1 & -1.1228 & -1.2180 & -0.8401 \\
2 & -0.7150 & -0.8362 & -0.5988 \\
3 & -1.6367 & -1.5413 & -1.5261 \\
4 & -1.3593 & -1.2041 & -1.3267 \\
5 & -1.7554 & -1.8701 & -1.6846 \\
6 & -2.1226 & -2.3514 & -1.9385 \\
7 & -2.2929 & -2.3568 & -2.3579 \\
8 & -2.8023 & -2.9306 & -2.8663 \\
9 & -3.0445 & -2.9943 & -2.9719 \\
10 & -3.4889 & -3.3824 & -2.9750 \\
11 & -3.4794 & -3.5896 & -3.3237 \\
12 & -3.4635 & -3.7971 & -3.5398 \\
13 & -3.6888 & -4.1857 & -3.7955 \\
14 & -3.8448 & -4.1124 & -3.9549 \\
15 & -4.0945 & -4.3040 & -4.0928 \\
16 & -4.0015 & -4.2972 & -4.0927 \\
17 & -3.9952 & -4.1224 & -4.0040 \\
18 & -4.0168 & -4.1569 & -4.1108 \\
19 & -4.0383 & -4.1908 & -4.2306 \\
20 & -4.2002 & -4.1429 & -4.3419 \\
21 & -4.1040 & -4.3728 & -4.3479 \\
22 & -4.1218 & -4.2493 & -4.1209 \\
23 & -4.1219 & -4.2553 & -3.9249 \\
24 & -4.3303 & -4.2030 & -4.1561 \\
25 & -4.1947 & -4.2743 & -4.1720 \\
26 & -4.2986 & -4.2314 & -4.1753 \\
27 & -4.0887 & -4.1126 & -4.1933 \\
28 & -4.0956 & -3.9658 & -4.0411 \\
29 & -4.1652 & -4.0173 & -4.0393 \\
30 & -4.1568 & -4.0311 & -4.0897 \\
31 & -4.1389 & -4.1134 & -4.0677 \\
32 & -4.1234 & -4.0914 & -4.0499 \\
33 & -4.1076 & -4.0932 & -4.0833 \\
34 & -3.8679 & -4.0807 & -4.0476 \\
35 & -3.9078 & -4.0378 & -4.0585 \\
36 & -4.2453 & -3.9664 & -3.9836 \\
37 & -3.7742 & -3.9499 & -3.9635 \\
38 & -3.9052 & -3.9963 & -4.0322 \\
39 & -3.7590 & -3.9894 & -4.0615 \\
40 & -3.9687 & -3.9451 & -3.9964 \\
\hline & & & \\
\hline & & &
\end{tabular}

This table lists the out-of-sample certainty equivalent return $(\widehat{C E})$ of the efficient expected utility/cardinality portfolios. MV refers to the efficient MV expected utility/cardinality portfolios, MVS refers to the efficient MVS expected utility/cardinality portfolios and MVSK refers to the efficient MVSK expected utility/cardinality portfolios. All the certainty equivalent values are multiplied by a factor of 1000 .

From the results in Table 17 we can see that, similarly to what occurred with PSI20 dataset, the certainty equivalent does not show a clear trend with increasing cardinality, and the best certainty equivalent values always occur at a low cardinality level (cardinality 2 for all frameworks). 
Table 18 reports the bootstrap $p$-values (computed according the Ledoit and Wolf, 2008 robust methodology) of the difference between the out-of-sample Sharpe ratio of each pair of frameworks (MV vs MVS, MV vs MVSK, and MVS vs MVSK) for the same cardinality level. Table 18 also reports, for each cardinality level, which framework shows the highest Sharpe ratio. For most cardinality levels, either the MVS or the MVSK efficient portfolios outperform the efficient MV portfolios, but such differences are never statistically significant.

The results for the out-of-sample certainty equivalent and Sharpe ratio, both suggest gains, for certain cardinality levels, in considering higher moments. These results are in accordance with the results obtained for the PSI20 dataset. 
Table 18: Bootstrap p-values for the Sharpe ratios, for the EUROSTOXX50 dataset

\begin{tabular}{|c|c|c|c|}
\hline Efficient portfolios & \multicolumn{3}{|c|}{ Bootstrap $p$-values } \\
\hline Number of stocks & MV vs MVS & MV vs MVSK & MVS vs MVSK \\
\hline 1 & 0.8018 (MVS) & 0.3827 (MVSK) & 0.3101 (MVSK) \\
\hline 2 & 0.5583 (MVS) & 0.4371 (MVSK) & 0.2486 (MVSK) \\
\hline 3 & 0.5211 (MV) & 0.7361 (MVSK) & 0.8246 (MVSK) \\
\hline 4 & 0.4119 (MV) & 0.9116 (MVSK) & 0.5945 (MVS) \\
\hline 5 & 0.6313 (MVS) & 0.8864 (MVSK) & 0.3275 (MVSK) \\
\hline 6 & 0.4409 (MVS) & 0.2242 (MVSK) & 0.0058 (MVSK) \\
\hline 7 & 0.6443 (MVS) & $0.5523(\mathrm{MV})$ & 0.8484 (MVS) \\
\hline 8 & 0.7281 (MVS) & 0.4811 (MV) & 0.5559 (MVSK) \\
\hline 9 & 0.5077 (MV) & 0.6737 (MVSK) & 0.6775 (MVSK) \\
\hline 10 & $0.6395(\mathrm{MV})$ & 0.0772 (MVSK) & 0.0940 (MVSK) \\
\hline 11 & 0.7806 (MVS) & 0.6211 (MVSK) & 0.4017 (MVSK) \\
\hline 12 & 0.1296 (MVS) & $0.4769(\mathrm{MV})$ & 0.3759 (MVSK) \\
\hline 13 & 0.0636 (MVS) & $0.4425(\mathrm{MV})$ & 0.2064 (MVSK) \\
\hline 14 & 0.2040 (MVS) & $0.3207(\mathrm{MV})$ & 0.7620 (MVSK) \\
\hline 15 & 0.5417 (MVS) & 0.9442 (MVSK) & 0.5455 (MVSK) \\
\hline 16 & 0.1772 (MVS) & $0.5427(\mathrm{MV})$ & 0.3493 (MVSK) \\
\hline 17 & 0.5688 (MVS) & 0.6474 (MVSK) & 0.8520 (MVSK) \\
\hline 18 & 0.5113 (MVS) & $0.5085(\mathrm{MV})$ & 0.9474 (MVSK) \\
\hline 19 & 0.5303 (MVS) & $0.2328(\mathrm{MV})$ & 0.5893 (MVS) \\
\hline 20 & $0.6087(\mathrm{MV})$ & 0.2346 (MV) & 0.0310 (MVS) \\
\hline 21 & 0.2791 (MVS) & $0.0963(\mathrm{MV})$ & 0.5367 (MVSK) \\
\hline 22 & 0.7271 (MVS) & 0.6437 (MVSK) & 0.9012 (MVSK) \\
\hline 23 & 0.9340 (MVS) & 0.3761 (MVSK) & 0.2244 (MVSK) \\
\hline 24 & $0.2460(\mathrm{MV})$ & 0.6237 (MVSK) & 0.4115 (MVSK) \\
\hline 25 & 0.8732 (MVS) & 0.8746 (MVSK) & 0.9968 (MVSK) \\
\hline 26 & 0.5815 (MV) & 0.6839 (MVSK) & 0.8890 (MVSK) \\
\hline 27 & 0.8546 (MVS) & $0.4657(\mathrm{MV})$ & 0.4809 (MVS) \\
\hline 28 & 0.3017 (MV) & 0.8048 (MVSK) & 0.3069 (MVS) \\
\hline 29 & 0.1588 (MV) & 0.3917 (MVSK) & 0.5469 (MVS) \\
\hline 30 & $0.2401(\mathrm{MV})$ & 0.8660 (MVSK) & 0.1978 (MVS) \\
\hline 31 & 0.4591 (MV) & 0.8498 (MVSK) & 0.4647 (MVSK) \\
\hline 32 & 0.3927 (MV) & 0.6527 (MVSK) & 0.6903 (MVSK) \\
\hline 33 & $0.4287(\mathrm{MV})$ & 0.9848 (MVSK) & 0.3715 (MVSK) \\
\hline 34 & 0.3655 (MVS) & $0.2334(\mathrm{MV})$ & 0.6139 (MVSK) \\
\hline 35 & 0.5713 (MVS) & $0.0819(\mathrm{MV})$ & 0.1556 (MVS) \\
\hline 36 & 0.1554 (MV) & 0.3589 (MVSK) & 0.3579 (MVS) \\
\hline 37 & 0.4453 (MVS) & $0.1414(\mathrm{MV})$ & 0.3659 (MVS) \\
\hline 38 & 0.9948 (MVS) & 0.6247 (MV) & 0.3119 (MVS) \\
\hline 39 & 0.2218 (MVS) & $0.0321(\mathrm{MV})$ & 0.1838 (MVS) \\
\hline 40 & 0.6309 (MVS) & $0.5311(\mathrm{MV})$ & 0.1858 (MVS) \\
\hline
\end{tabular}

This table lists the bootstrap p-values of the difference between the Sharpe ratio of each pair of frameworks. $M V$ refers to the efficient $M V$ expected utility/cardinality portfolios, MVS refers to the efficient MVS expected utility/cardinality portfolios, and MVSK refers to the efficient MVSK expected utility/cardinality portfolios. The bootstrap p-values were computed according the Ledoit and Wolf (2008) methodology. In parenthesis there is the indication of the framework with the highest Sharpe ratio. 
Similarly to what occurred with the PSI20 dataset, the turnover exhibits a non-linear pattern (see Table 19). In all the frameworks we observe that the turnover begins by increasing up to a certain cardinality level (17 in the EUROSTOXX50 dataset case, which corresponds to $42.5 \%$ of the maximum cardinality level), then decreases. However, starting from the cardinality level equal to 24 we observe, again, an increase in the turnover. Then, for high cardinality levels, the trend ceases to be clear. 
Table 19: Portfolio turnover, for the EUROSTOXX50 dataset

\begin{tabular}{|c|c|c|c|}
\hline \multicolumn{4}{|l|}{ Efficient portfolios } \\
\hline Number of stocks & MV & MVS & MVSK \\
\hline 1 & 13.91 & 13.83 & 15.03 \\
\hline 2 & 13.13 & 14.68 & 15.07 \\
\hline 3 & 40.13 & 44.38 & 49.43 \\
\hline 4 & 56.03 & 60.37 & 64.73 \\
\hline 5 & 50.12 & 60.78 & 55.56 \\
\hline 6 & 80.35 & 78.80 & 83.38 \\
\hline 7 & 84.60 & 82.01 & 87.81 \\
\hline 8 & 81.93 & 97.10 & 102.55 \\
\hline 9 & 72.52 & 96.75 & 97.60 \\
\hline 10 & 107.11 & 130.02 & 130.80 \\
\hline 11 & 133.03 & 132.08 & 144.80 \\
\hline 12 & 138.50 & 142.45 & 148.69 \\
\hline 13 & 138.05 & 150.00 & 146.92 \\
\hline 14 & 141.36 & 138.21 & 141.51 \\
\hline 15 & 137.17 & 137.64 & 148.30 \\
\hline 16 & 139.56 & 143.85 & 149.47 \\
\hline 17 & 141.55 & 141.74 & 153.49 \\
\hline 18 & 126.50 & 131.27 & 136.05 \\
\hline 19 & 125.16 & 124.90 & 129.02 \\
\hline 20 & 123.98 & 126.50 & 127.59 \\
\hline 21 & 125.75 & 120.89 & 122.24 \\
\hline 22 & 118.06 & 120.93 & 122.45 \\
\hline 23 & 117.59 & 119.51 & 121.68 \\
\hline 24 & 118.82 & 122.29 & 130.72 \\
\hline 25 & 127.28 & 134.03 & 135.81 \\
\hline 26 & 128.94 & 135.64 & 136.83 \\
\hline 27 & 129.05 & 136.34 & 139.51 \\
\hline 28 & 136.11 & 139.21 & 140.43 \\
\hline 29 & 135.31 & 137.47 & 143.29 \\
\hline 30 & 139.45 & 139.46 & 143.51 \\
\hline 31 & 136.33 & 141.39 & 141.24 \\
\hline 32 & 136.19 & 140.40 & 143.50 \\
\hline 33 & 133.07 & 141.99 & 142.56 \\
\hline 34 & 141.78 & 142.74 & 141.08 \\
\hline 35 & 136.67 & 140.69 & 143.49 \\
\hline 36 & 166.34 & 142.33 & 142.41 \\
\hline 37 & 145.50 & 138.73 & 144.47 \\
\hline 38 & 166.21 & 160.99 & 157.54 \\
\hline 39 & 145.69 & 139.39 & 146.63 \\
\hline 40 & 137.55 & 139.72 & 141.86 \\
\hline
\end{tabular}

This table lists the portfolio turnover of the efficient expected utility/cardinality portfolios. MV refers to the efficient MV expected utility/cardinality portfolios, MVS refers to the efficient MVS expected utility/cardinality portfolios and MVSK refers to the efficient MVSK expected utility/cardinality portfolios. 
Regarding the net Sharpe ratio (see Table 20), as for the PSI20 dataset, we observe that sparse portfolios tend to exhibit the best performance: in all the three frameworks the efficient portfolios corresponding to the cardinality level equal 2 are the portfolios with the highest refined net Sharpe ratio. Finally, we notice that, for the EUROSTOXX50 dataset, the MV efficient portfolios outperform the MVS and the MVSK efficient portfolios for most cardinalities. 
Table 20: Refined Sharpe ratios of returns net of transaction costs, for the EUROSTOXX50 dataset

\begin{tabular}{c|c|c|c}
\hline \multicolumn{4}{c}{ Efficient portfolios } \\
\hline \hline Number of stocks & MV & MVS & MVSK \\
\hline 1 & -0.1360 & -0.1395 & -0.1426 \\
2 & -0.1073 & -0.1237 & -0.1213 \\
3 & -0.4877 & -0.5414 & -0.5870 \\
4 & -0.6547 & -0.7181 & -0.7536 \\
5 & -0.6448 & -0.7818 & -0.7024 \\
6 & -1.0447 & -1.0544 & -1.0964 \\
7 & -1.1716 & -1.1325 & -1.2033 \\
8 & -1.2258 & -1.4757 & -1.4913 \\
9 & -1.0991 & -1.5041 & -1.4862 \\
10 & -1.6402 & -2.0026 & -1.9751 \\
11 & -2.0884 & -2.0941 & -2.2504 \\
12 & -2.2377 & -2.2704 & -2.3405 \\
13 & -2.2362 & -2.4233 & -2.3268 \\
14 & -2.3225 & -2.2756 & -2.2848 \\
15 & -2.2672 & -2.2993 & -2.4405 \\
16 & -2.3264 & -2.3787 & -2.4668 \\
17 & -2.3837 & -2.3738 & -2.5310 \\
18 & -2.1571 & -2.2256 & -2.2903 \\
19 & -2.1514 & -2.1606 & -2.2140 \\
20 & -2.1534 & -2.2148 & -2.1834 \\
21 & -2.2019 & -2.1345 & -2.1065 \\
22 & -2.0808 & -2.1540 & -2.1216 \\
23 & -2.0638 & -2.1430 & -2.1198 \\
24 & -2.1094 & -2.2029 & -2.2845 \\
25 & -2.2693 & -2.4090 & -2.3981 \\
26 & -2.3133 & -2.4471 & -2.4359 \\
27 & -2.3214 & -2.4474 & -2.4920 \\
28 & -2.4312 & -2.5182 & -2.5045 \\
29 & -2.4173 & -2.4838 & -2.5619 \\
30 & -2.4952 & -2.5202 & -2.5465 \\
31 & -2.4408 & -2.5786 & -2.5069 \\
32 & -2.4298 & -2.5431 & -2.5554 \\
33 & -2.3817 & -2.5873 & -2.5390 \\
34 & -2.5413 & -2.5894 & -2.5184 \\
35 & -2.4547 & -2.5517 & -2.5375 \\
36 & -2.9631 & -2.5736 & -2.5236 \\
37 & -2.6060 & -2.5100 & -2.5616 \\
38 & -2.9530 & -2.9100 & -2.7977 \\
39 & -2.6082 & -2.5201 & -2.6066 \\
40 & -2.4691 & -2.5253 & -2.5195 \\
\hline & & & \\
\hline
\end{tabular}

This table reports the refined Sharpe ratios of returns net of transaction costs for each efficient expected utility/cardinality portfolio. MV refers to the efficient MV expected utility/cardinality portfolios, MVS refers to the efficient MVS expected utility/cardinality portfolios and MVSK refers to the efficient MVSK expected utility/cardinality portfolios. The refined net Sharpe ratios were computed according the Israelsen (2005) methodology. 


\section{Conclusions}

This paper extends the analysis of the cardinality impact in the portfolio performance from the standard mean-variance framework to frameworks with higher moments, namely by considering the skewness and kurtosis. We propose a bi-objective model that allows the direct analysis of the efficient tradeoff between expected utility and cardinality. This analysis was conducted assuming an investor with CRRA preferences (with a relative risk aversion parameter of five) and within mean-variance, mean-variance-skewness and mean-variance-skewness-kurtosis frameworks. Although we have assumed a specific form for the investor's utility, the proposed methodology may easily be applied to any other utility type function.

We have conducted an empirical application of the proposed methodology to a dataset based on the Portuguese Stock Index (PSI20 index). We also used a dataset based on the EUROSTOXX50 index in order to check the robustness of those results. The results showed that both the in-sample certainty equivalent and the in-sample Sharpe ratio increase with cardinality. The empirical analysis also suggests that there are no performance gains in-sample (in terms of certainty equivalent) when higher moments are considered. On the other hand, the out-of-sample certain equivalent and the out-of-sample Sharpe ratio show no clear trend with increasing cardinality, when transaction costs are ignored.

The portfolio turnover presents a non-linear trend, increasing up to a certain cardinality level, and then decreasing. In the case of the EUROSTOXX50, it afterwards increases again, and shows an unclear trend for larger cardinality levels. This implies that diversification is an important issue in portfolio management when transaction costs are considered, and therefore when another degree of reality is brought into the analysis.

Finally, the out-of-sample comparison of different frameworks allows the conclusion that, in some cases, the inclusion of higher moments in the portfolio management framework may lead to some significant gains in terms of certainty equivalent and Sharpe ratio. However, this result seems to be quite sensitive to the dataset and to the cardinality level.

In this study we assumed that the investor has CRRA preferences with a relative risk aversion of five. In future research we are also interested in studying the sensitivity of these results to different choices of the relative risk aversion level and also to different utility type functions (e.g. HARA utility).

\section{References}

Ait-Sahalia, Y. and M. W. Brandt (2001). Variable selection for portfolio choice. Journal of Finance 56, $1297-1351$.

Anagnostopoulos, K. P. and G. Mamanis (2011). The mean-variance cardinality constrained portfolio optimization problem: An experimental evaluation of five multiobjective evolutionary algorithms. Expert Systems with Applications 38, 14208-14217.

Arditti, F. D. (1967). Risk and the required return on equity. The Journal of Finance 22, 19-36.

Athayde, G. and R. Flôres (2004). Finding a maximum skewness portfolio: A general solution to threemoments portfolio choice. Journal of Economic Dynamics \& Control 28, 1335-1352.

Bai, X., J. R. Russell, and G. C. Tiao (2003). Kurtosis of GARCH and stochastic volatility models with non-normal innovations. Journal of Econometrics 114, 349-360. 
Benartzi, S. and R. H. Thaler (2001). Naive diversification strategies in defined contribution saving plans. The American Economic Review 91, 79-98.

Bertsimas, D. and R. Shioda (2009). Algorithm for cardinality-constrained quadratic optimization. Comput. Optim. Appl. 43, 1-22.

Bienstock, D. (1996). Computational study of a family of mixed-integer quadratic programming problems. Math. Program. 74, 121-140.

Bollerslev, T. (1986). Generalized autoregressive conditional heteroskedasticity. Journal of Econometrics 31, 307-327.

Brandt, M. W., P. Santa-Clara, and R. Valkanov (2009). Parametric portfolio policies: Exploiting characteristics in the cross-section of equity returns. Review of Financial Studies 22, 3411-3447.

Brito, R. P. and L. N. Vicente (2014). System Modeling and Optimization: 26th IFIP TC 7 Conference, CSMO 2013, Klagenfurt, Austria, September 9-13, 2013, Revised Selected Papers, Chapter Efficient Cardinality/Mean-Variance Portfolios, pp. 52-73. Springer Berlin Heidelberg.

Brodie, J., I. Daubechies, C. D. Mol, D. Giannone, and I. Loris (2009). Sparse and stable Markowitz portfolios. Proc. Natl. Acad. Sci. USA 106, 12267-12272.

Cesarone, F., A. Scozzari, and F. Tardella (2009). Efficient algorithms for mean-variance portfolio optimization with hard real-world constraints. Giornale dell'Istituto Italiano degli Attuari 72, 37-56.

Chang, T. J., N. Meade, J. E. Beasley, and Y. M. Sharaiha (2000). Heuristics for cardinality constrained portfolio optimisation. Comput. Oper. Res. 27, 1271-1302.

Chunhachinda, P., K. Dandapani, S. Hamid, and A. J. Prakash (1997). Portfolio selection and skewness: Evidence from international stock markets. Journal of Banking $\&$ Finance 21, 143-167.

Conn, A. R., K. Scheinberg, and L. Vicente (2009). Introduction to Derivative-Free Optimization. Philadelphia: MPS-SIAM Series on Optimization.

Cremers, J. H., M. Kritzman, and S. Page (2005). Optimal hedge fund allocations - Do higher moments matter? Journal of Portfolio Management 3, 70-81.

Curto, J., E. Reis, and J. Esperança (2003). Stable paretian distributions: an unconditional model for psi20, dax and djia indexes. Review of Financial Markets 5, 5-18.

Custódio, A. L., J. F. A. Madeira, A. I. F. Vaz, and L. N. Vicente (2011). Direct multisearch for multiobjective optimization. SIAM J. Optim. 21, 1109-1140.

DeMiguel, V., L. Garlappi, F. J. Nogales, and R. Uppal (2009). A generalized approach to portfolio optimization: Improving performance by constrained portfolio norms. Management Science 55, 798812 .

DeMiguel, V., F. J. Nogales, and R. Uppal (2014). Stock return serial dependence and out-of-sample portfolio performance. The Review of Financial Studies 27, 1031-1073.

DeMiguel, V. and A. V. Olivares-Nadal (2015). A robust perspective on transaction costs in portfolio optimization. Working paper, London Business School.

Efron, B. and R. J. Tibshirani (1994). An Introduction to the Bootstrap. Chapman \& Hall/CRC Monographs on Statistics \& Applied Probability. Taylor \& Francis. 
Engle, R. F. (1982). Autoregressive conditional heteroskedasticity with estimates of the variance of u.k. inflation. Econometrica 50, 987-1008.

Evans, J. L. and S. H. Archer (1968). Diversification and the reduction of dispersion: An empirical analysis. Journal of Finance 23, 761-767.

Fama, E. (1965). The behavior of stock-market prices. The Journal of Business 38, 34-105.

Fieldsend, J. E., J. Matatko, and M. Peng (2004). Cardinality constrained portfolio optimisation. In Intelligent Data Engineering and Automated Learning - IDEAL 2004, Volume 3177 of Lecture Notes in Computer Science, pp. 788-793. Berlin: Springer.

Harvey, C. R., J. Liechty, M. W. Liechty, and P. Mueller (2010). Portfolio selection with higher moments. Quantitative Finance 10, 469-485.

Harvey, C. R. and A. Siddique (2000). Conditional skewness in asset pricing tests. The Journal of Finance 55, 1263-1295.

Israelsen, C. L. (2005). A refinement to the sharpe ratio and information ratio. Journal of Asset Management 5, 423-427.

Jobst, N. J., M. D. Hornimam, C. A. Lucas, and G. Mitra (2010). Computational aspects of alternative portfolio selection models in the presence of discrete asset choice constraints. Quantitative Finance 1, 489-501.

Kraus, A. and R. H. Litzenberger (1976). Skewness preference and the valuation of risk assets. The Journal of Finance 31, 1085-1100.

Ledoit, O. and M. Wolf (2008). Robust performance hypothesis testing with the Sharpe ratio. Journal of Empirical Finance 15, 850-859.

Mandelbrot, B. (1963). The variation of certain speculative prices. The Journal of Business 36, 394.

Maringer, D. and P. Parpas (2009). Global optimization of higher order moments in portfolio selection. J. Global Optim. 43, 219-230.

Markowitz, H. M. (1952). Portfolio selection. The Journal of Finance 7, 77-91.

Mencia, J. and E. Sentana (2009). Multivariate location-scale mixtures of normals and mean-varianceskewness portfolio allocation. Journal of Econometrics 153, 105-121.

Michaud, R. O. (1989). The markowitz optimization enigma: Is optimized optimal? Financial Analysts Journal 4, 31-42.

Nelson, D. B. (1991). Conditional heteroskedasticity in asset returns: a new approach. Econometrica 59, $347-370$

Rege, S., J. C. A. Teixeira, and A. G. Menezes (2013). The daily returns of the portuguese stock index: a distributional characterization. Journal of Risk Model Validation 7, 53-70.

Statman, M. (1987). How many stocks make a diversified portfolio? Journal of Financial and Quantitative Analysis 22, 353-363.

Tavares, A. B., J. D. Curto, and G. N. Tavares (2008). Modelling heavy tails and asymmetry using arch-type models with stable paretian distributions. Nonlinear Dynamics 51, 231-243.

Taylor, S. J. (1986). Modelling Finantial Time Series. Chichester, UK: Wiley. 
Turner, A. L. and E. J. Weigel (1992). Daily stock market volatility: 1928-1989. Management Science 38, 1586-1609.

Vielma, J. P., S. Ahmed, and G. L. Nemhauser (2008). A lifted linear programming branch-and-bound algorithm for mixed-integer conic quadratic programs. INFORMS J. Comput. 20, 438-450.

Woodside-Oriakhi, M., C. Lucas, and J. E. Beasley (2011). Heuristic algorithms for the cardinality constrained efficient frontier. European J. Oper. Res. 213, 538-550.

Zakoian, J. M. (1994). Threshold heteroskedastic models. Journal of Economic Dynamics and Control 18, 931-955. 